\title{
Casiopeina II-gly acts on IncRNA MALAT1 by miR-17-5p to inhibit FZD2 expression via the Wnt signaling pathway during the treatment of cervical carcinoma
}

\author{
YUNSHENG XU ${ }^{1,2}$, QIANWEN ZHANG ${ }^{3}$, FAN LIN $^{3}$, LI ZHU $^{4}$, FANGFANG HUANG ${ }^{4}$, \\ LIANG $\mathrm{ZHAO}^{5}$ and RONGYING OU ${ }^{4}$ \\ ${ }^{1}$ Laboratory for Advanced Interdisciplinary Research, Institutes of Translational Medicine, \\ The First Affiliated Hospital of Wenzhou Medical University, Wenzhou, Zhejiang 325000; \\ ${ }^{2}$ Department of Dermatovenereology, The Seventh Affiliated Hospital, Sun Yat-sen University, \\ Shenzhen, Guangdong 518107; Departments of ${ }^{3}$ Dermatovenereology, ${ }^{4}$ Gynaecology and Obstetrics and \\ ${ }^{5}$ Laboratory for Advanced Interdisciplinary Research, The First Affiliated Hospital of \\ Wenzhou Medical University, Wenzhou, Zhejiang 325000, P.R. China
}

Received August 15, 2018; Accepted January 22, 2019

DOI: $10.3892 / o r .2019 .7268$

\begin{abstract}
The present study investigated the underlying regulatory network involved in the differential expression of metastasis associated lung adenocarcinoma transcript 1 (MALAT1) long non-coding (lnc)RNA, microRNA-17-5p (miR-17-5p) and frizzled class receptor 2 (FZD2) mRNA under the influence of Casiopeina II-gly (Cas-II-gly) via the Wnt signaling pathway in cervical carcinoma (CC). The gene expression data were obtained from the Gene Expression Omnibus database (https://www.ncbi.nlm.nih.gov/geo/), and the differentially expressed genes were determined using R software. The R ClusterProfiler and enrichplot packages were applied for gene-set enrichment analysis based on the Gene Ontology biological process and Kyoto Encyclopedia of Genes and Genomes databases. TargetScan and the starBase database were used to predict the targeting
\end{abstract}

Correspondence to: Dr Rongying Ou, Department of Gynaecology and Obstetrics, The First Affiliated Hospital of Wenzhou Medical University, 12 Wendi Road, Wenzhou, Zhejiang 325000, P.R. China E-mail: ourongying@163.com

Dr Yunsheng Xu, Department of Dermatovenereology, The Seventh Affiliated Hospital, Sun Yat-sen University, 628 Dongyuan Road, Shenzhen, Guangdong 518107, P.R. China

E-mail: xuyunsheng1018@163.com

Abbreviations: Cas-II-gly, Casiopeina II-gly; CC, cervical cancer; DEGs, differentially-expressed genes; FBS, fetal bovine serum; GO, Gene Ontology; GSEA, gene set enrichment analysis; lncRNAs, long non-coding RNAs; PI3K, phosphoinositide 3-kinase; RT, reverse transcription

Key words: Casiopeina II-gly, metastasis associated lung adenocarcinoma transcript 1, frizzled class receptor 2, microRNA-17-5p, Wnt signaling pathway, cervical carcinoma associations between the miRNAs and lncRNAs/mRNAs. The MALAT1/miR-17-5p/mRNA FZD2 expression levels were measured via reverse transcription-quantitative polymerase chain reaction. The protein expression was monitored by western blot analysis. The target association among the lncRNA MALAT1, miR-17-5p and FZD2 was validated via a dual luciferase reporter assay. Cell viability and apoptosis were determined via MTT assays, EdU staining and flow cytometry. The results indicated that the expression levels of IncRNA MALAT1 and FZD2 mRNA were downregulated, while miR-17-5p expression was upregulated in HeLa and CaSki cells treated with increasing Cas-II-gly concentrations. The cell viability was decreased, and the apoptosis rate was increased in HeLa and CaSki cells following Cas-II-gly treatment. Furthermore, western blot analysis results demonstrated that Cas-II-gly and the MALAT1/miR-17-5p/FZD2 axis could affect the expression of proteins associated with the Wnt signaling pathway, including disheveled segment polarity protein, glycogen synthase kinase- $3 \beta$ and $\beta$-catenin, and via the MALAT1/miR-17-5p/FZD2/Wnt signaling pathway axis.

\section{Introduction}

Cervical cancer (CC) is the second leading cause of cancer-associated mortalities among women aged 20-39 years old in USA in 2015, and it should receive attention due to the requirement for preventing the disease in young women (1). Chronic infection with human papillomavirus (HPV) can result in cervical intraepithelial neoplasia and deterioration of the cervix (2). Expression of the viral proteins E6 and E7 is involved in CC, and the former can enhance the degradation of the p53 tumor suppressor protein, whereas the latter serves key roles in the HPV life cycle and carcinogenic transformation $(3,4)$. The comprehensive mechanism underlying $\mathrm{CC}$ still remains to be fully clarified.

Casiopeina II-gly (Cas-II-gly) is a novel chemotherapeutic compound based on a copper core, and it is an efficient antitumor agent for carcinoma cell lines in in vitro experiments (5). 
A number of studies indicated that Cas-II-gly could involve interaction with DNA, via intercalation or direct adduct formation (6), or poisoning and oxidation of mitochondrion (7). Additionally, a previous report demonstrated that Cas-II-gly can significantly suppress HeLa cell proliferation (8); however, its mechanism remains unclear. The present study focuses on the effects of Cas-II-gly on gene expression and cellular functions in HeLa and CaSki CC cells.

Microarray analysis is an effective method to examine specific genes and their properties among thousands of samples (9-11). Almost all differentially-expressed genes (DEGs) are divided into three major categories based on Gene Ontology (GO) annotations: Biological processes; cellular components; and molecular functions (12). A number of studies have been performed using this approach. For example, using a microRNA (miRNA) microarray, Xia et al (13) determined that miR-3156-3p expression was significantly suppressed in CC cells, compared with the control cells. In another study, Li et al (14) derived data from The Cancer Genome Atlas database and identified 11 upregulated and 28 downregulated DEGs in CC, as well as 5 significantly-enriched pathways being validated in the ClueGO and DAVID databases, including the systemic lupus erythematosus pathway (15). Liang et al (16) employed functional enrichment analysis to demonstrate that the target genes of three miRNAs (miRNA-145/200c/218-1) may participate in diverse pathways in $\mathrm{CC}$, including the mitogen-activated protein kinase (MAPK), adenosine 5'-monophosphate-activated protein kinase, focal adhesion, cGMP-protein kinase G, Wnt and mechanistic target of rapamycin kinase signaling pathways, which have significant impacts on CC cells (16). In another study, Liang et al (17) determined that miR-101 (screened out using 3 bioinformatics web sites) suppressed the G1-to-S phase transition in CC cells. All of these examples demonstrate that microarray analysis is a powerful and effective method that can help to identify the underlying genes and signaling pathways responsible for diseases. However, the underlying regulatory networks involve greater complexity that remains to be comprehensively dissected. The aim of the present study was to analyze the DEGs between untreated CC cells and CC cells treated with Cas-II-gly.

Long non-coding (lnc)RNAs regulate the growth, invasion and deterioration of tumors (18). The results of Fan et al (19) demonstrated that upregulation of lnc-interleukin 7 receptor could reduce tumor size and limit lymph node metastasis in $\mathrm{CC}$ tissue. Another study indicated that transfection of $\mathrm{CC}$ cells with a small interfering (si)RNA against prostate cancer associated transcript-1 (PCAT-1) lncRNA could significantly suppress PCAT-1 expression, while PCAT-1 overexpression could limit the proliferation, metastasis and invasion of $\mathrm{CC}$ cells (20). miRNAs are a class of 18-25 nucleotide-long RNA molecules that are involved in maintaining normal cellular function by regulating mRNA transcription (21). For instance, miR-519d-3p overexpression inhibited cell proliferation and cell cycle progression, while it enhanced apoptosis in $\mathrm{HeLa}$ cells by targeting hypoxia inducible factor $1 \alpha$ (22). In another study, Cai et al (23) determined that by targeting transforming growth factor $\beta$ receptor 2 , miR-17-5p had a positive correlation with $\mathrm{CC}$ proliferation and metastasis. Recently, the IncRNA-miRNA-mRNA axis was assigned a broad role in the regulatory mechanisms of disease-associated genes, including genes involved in CC (12,24-27). Furthermore, the lncRNA-miRNA-mRNA axis also serves an important role in regulating genes involved in CC sensitivity to chemotherapy, including cancer susceptibility candidate 2-miR-21 and phosphatase and tensin homolog (28). Therefore, the present study also aimed to clarify the genetic basis of $\mathrm{CC}$, which is essential for investigating the underlying mechanism of Cas-II-gly in $\mathrm{CC}$.

The Wnt signaling pathway is a complex regulatory network that participates in cell differentiation, proliferation, migration, polarity, processes that are crucial for embryonic development, tissue regeneration, stem cell maintenance and homeostasis (29). For example, it has been reported that the Wnt/ $\beta$-catenin/Notch and phosphoinositide 3-kinase $(\mathrm{PI} 3 \mathrm{~K}) /$ protein kinase $\mathrm{B}(\mathrm{Akt})$ pathways are deregulated in HPV-induced cancer (30). The results from Li et al (31) indicated that by targeting the $A P C$ gene, miR-182 activated the canonical Wnt signaling pathway and increased $\mathrm{CC}$ cell proliferation. Those observations provided a molecular-level perspective on CC cell regulation. However, this network, which includes miRNAs, lncRNAs and the Wnt pathway, is complex and further studies are required to investigate the regulatory associations between the IncRNA-miRNA axis and the Wnt signaling pathway.

\section{Materials and methods}

Dataset. The data were harvested from the Gene Expression Omnibus database accession GSE41827 (https://www.ncbi. nlm.nih.gov/geo/query/acc.cgi?acc=GSE41827). A total of 6 samples were divided into three control HeLa groups and three Cas-II-gly treated HeLa groups (3 samples/group) in the microarray data (7). In the dataset, HeLa cells in the treatment group were exposed to Cas-II-gly $(40 \mathrm{mM})$ in 96 -well microplates for $6 \mathrm{~h}$ at $37^{\circ} \mathrm{C}$ and maintained at $37^{\circ} \mathrm{C}$ in $5 \% \mathrm{CO}_{2}$ under sterile conditions in Dulbecco's modified Eagle's medium supplemented with $10 \%$ fetal bovine serum (FBS), along with an untreated control group. The $\mathrm{R}$ (version 3.4.1) Limma package (32) was used to analyze the DEGs. $\mid \log _{2} \mathrm{FCl}>1$ and $\mathrm{P}<0.05$ served as the standard to identify DEGs. The probes of the DEGs were then replaced by the Gene Symbol listed in GPL570 (https://www.ncbi.nlm.nih.gov/geo/query/acc. cgi?acc $=$ GPL570).

Functional enrichment analysis. The GO (33) analysis consisted of the cellular composition, molecular function and biological process. The ClusterProfiler (http://www.bioconductor.org/packages/release/bioc/html/clusterProfiler.html) package was used to perform the GO biological process functional enrichment analysis of the relevant DEGs, and the hypergeometric distribution method (34) was used to calculate the $\mathrm{P}$-value. $\mathrm{P}<0.05$ served as the threshold for statistical significance. The results of ClusterProfiler enrich analysis were visualized via the enrichplot (https://github.com/GuangchuangYu/enrichplot) and cnetplot (34) package.

Pathway enrichment analysis. Based on the Kyoto Encyclopedia of Genes and Genomes database, which includes systems information, genomic information and chemicalinformation(35), gene 
set enrichment analysis was conducted via the ClusterProfiler package to identify statistically significant signaling pathways $(\mathrm{P}<0.05)$. The visualization was produced using the enrichplot package.

Correlation network analysis among the DEGs. To identify the correlation between mRNAs and IncRNAs, the psych package (https://cran.rstudio.com/web/packages/psych/index. html) was used to establish a correlation network, which was built according to the normalized expression levels of the lncRNAs and mRNAs. Pearson's coefficient was calculated to form the network (36). Cutoff $>0.7$ and $P<0.05$ served as the threshold for statistical significance. The network was visualized via the Cytoscape software (version 3.6.0; http://www. cytoscape.org/).

miRNA target prediction. The TargetScan (http://www. targetscan.org/vert_72/) and starBase databases (http://starbase.sysu.edu.cn/) were employed to predict the targeting associations between miRNAs and lncRNAs/mRNAs. The Venny tool (http://bioinfogp.cnb.csic.es/tools/venny/) was applied to identify the common miRNAs among the predicted results.

Cell line cultivation. The CC cell lines [CaSki (cat. no. BNCC338223) and HeLa (cat. no. BNCC337633; BeNa Culture Collection, Beijing, China)] and the human cervical epithelial cell line HcerEpic (cat. no. BNCC340374; BeNa Culture Collection) were maintained at $37^{\circ} \mathrm{C}$ in $5 \% \mathrm{CO}_{2}$ under sterile conditions. The CaSki and HeLa cell lines were cultured in CM2-1 solution, which consisted of RPMI-1640 (Sigma-Aldrich; Merck KGaA; Darmstadt, Germany) and FBS (9:1 ratio). The HcerEpic cell line was cultured in Eagle's Minimum Essential medium (Minimum Essential medium and non-essential amino acid; Sigma-Aldrich; Merck KGaA) with FBS (9:1 ratio).

Plasmid construction, oligonucleotides and cell transfection. The MALAT1- and FZD2-encoding sequences were amplified by polymerase chain reaction (PCR), according to the subsequent protocol, and then cloned into a pcDNA3.1 vector (25 nM; Shanghai GenePharma Co., Ltd., Shanghai, China) for overexpression. MALAT1-siRNA (si-MALAT1; $50 \mathrm{nM}$ ) was used to target MALAT1 and FZD2-siRNA (si-FZD2; $50 \mathrm{nM}$ ) was used to knockdown $F Z D 2$, which were purchased from Shanghai GenePharma Co., Ltd. The sequences were as follows: si-MALAT1, 5'-CAGCCCGAGACTTCTGTAA-3'; and si-FZD2, 5'-CCCGACTTCACGGTCTACATGATCA-3'. The hsa-miR-17-5p mimic and inhibitor were also purchased from Shanghai GenePharma Co., Ltd. The oligonucleotides and constructs were transfected separately into cells using Lipofectamine ${ }^{\circledR} 2000$ (Invitrogen; Thermo Fisher Scientific, Inc., Waltham, MA, USA), according to the manufacturer's protocols. The subsequent experimentation was performed $24 \mathrm{~h}$ after transfection.

Synthesis of Cas-II-gly. The Cas-II-gly copper complex was synthesized. Firstly, equimolar solutions of copper (II) nitrate and the corresponding substituted diamine were mixed. Subsequently, a previously deprotonated N-O donor was added(37). The product was consistent with the characterization reported by Alemon-Medina et al (38) (Table SI).

Reverse transcription-quantitative PCR (RT-qPCR). RT-qPCR was used to monitor the amplification of target DNA molecules during the PCR in real time, which was used to estimate the expression levels of the RNAs in the present study. HeLa and CaSki cells were treated with different concentrations of Cas-II-gly $(0,10,20,30$ or $40 \mathrm{mM})$ for $6 \mathrm{~h}$ at $37^{\circ} \mathrm{C}$. Subsequently, total RNA was isolated using TRIzol ${ }^{\circledR}$ Reagent (Invitrogen; Thermo Fisher Scientific, Inc.), according to the manufacturer's protocols. miRNA-specific probes were designed using the PrimerExpress ${ }^{\circledR}$ V3.0.1 software (Applied Biosystems; Thermo Fisher Scientific, Inc.) and purchased from Integrated DNA Technologies (Intergrated DNA Technologies, Inc., Coralville, IA, USA). RT was performed using a Super-Script First-Strand Synthesis kit (Invitrogen; Thermo Fisher Scientific, Inc.). qPCR was performed using a standard TaqMan PCR kit protocol on an Applied Biosystems 7900HT Sequence Detection System (cat. no. 4329002; Applied Biosystems; Thermo Fisher Scientific, Inc.). The PCR reaction mixture included the RT product, 2X TaqMan ${ }^{\circledR}$ Universal PCR Master Mix (cat. no. 4324018), $0.2 \mu \mathrm{M}$ TaqMan ${ }^{\circledR}$ probe (cat. no. 4316033 ; both Applied Biosystems; Thermo Fisher Scientific, Inc.), $1.5 \mu \mathrm{M}$ forward primer and $0.7 \mu \mathrm{M}$ reverse primer. The thermocycling conditions for the PCR were as follows: Preheating at $90^{\circ} \mathrm{C}$ for $10 \mathrm{~min}$, followed by 30 cycles of $95^{\circ} \mathrm{C}$ for $30 \mathrm{sec}, 55^{\circ} \mathrm{C}$ for $30 \mathrm{sec}$, $72^{\circ} \mathrm{C}$ for $60 \mathrm{sec}$, with a final elongation step at $72^{\circ} \mathrm{C}$ for $10 \mathrm{~min}$ and $4^{\circ} \mathrm{C}$ on hold. Following the completion of the reactions, the $\mathrm{Cq}$ values were determined using the default threshold settings. The ratio of mRNA to IncRNA was calculated by using the $2^{-\Delta \Delta \mathrm{Cq}}$ equation (39). The sequences are listed in Table I.

Western blot analysis. Western blot analysis was conducted to evaluate protein expression levels. Following transfection in HeLa and CaSki cells, according to the aforementioned protocol, proteins were extracted in lysis buffer (Sigma-Aldrich; Merck $\mathrm{KGaA}$ ), boiled for $5 \mathrm{~min}$ at $100^{\circ} \mathrm{C}$ and then cooled on ice for $5 \mathrm{~min}$. Protein concentrations were quantified by the bicinchoninic acid protein assay. Subsequently, $30 \mu \mathrm{g}$ total protein was resolved via $12 \%$ SDS-PAGE and the transferred to a polyvinylidene difluoride membrane (EMD Millipore, Billerica, MA, USA). The membranes were blocked in $5 \%$ fat-free milk in TBST buffer $(50 \mathrm{mM}$ Tris- $\mathrm{HCl}, 150 \mathrm{mM}$ $\mathrm{NaCl}$ and $0.05 \%$ Tween-20; $\mathrm{pH} 7.6$ ) at $4^{\circ} \mathrm{C}$ for $1 \mathrm{~h}$. Subsequently, the membranes were incubated with anti-FZD2 (1:2,000; cat. no. ab94913), anti-disheveled segment polarity protein (Dvl; 1:2,000; cat. no. ab233003), anti-glycogen synthase kinase-3 $\beta$ (GSK-3 $\beta$; 1:5,000; cat. no. ab32391), anti- $\beta$-catenin (1:5,000; cat. no. ab32572) and anti-GAPDH (1:2,500; cat. no. ab9485) primary antibodies at $4^{\circ} \mathrm{C}$ overnight. Following three washes in TBST buffer, the membranes were incubated with the goat anti-rabbit horseradish peroxidase-conjugated immunoglobulin G secondary antibody $(1: 2,000$; cat. no. ab6721) at room temperature for $1 \mathrm{~h}$ and then washed three times in TBST buffer. The blots were visualized by intense chemiluminescence detection (GE Healthcare Life Sciences, Little Chalfont, UK) and exposed to Kodak XAR-5-ray film (Sigma-Aldrich; Merck KGaA). All antibodies were purchased from Abcam (Cambridge, MA, USA). 
Table I. Primer sequences used for reverse transcriptionquantitative polymerase chain reaction.

\begin{tabular}{ll} 
Genes & \multicolumn{1}{c}{ Primer sequences (5'-3') } \\
\hline MALAT1 & F: AAAGCAAGGTCTCCCCACAAG \\
R: GGTCTGTGCTAGATCAAAAGGC \\
F: GCGAAGCCCTCATGAACAAG \\
RiR-17-5p & F: CAAAGTGCTTACAGTGCAGGTAG \\
& R: GAATACCTCGGACCCTGC \\
U6 & F: CTCGCTTCGGCAGCACA \\
& R: AACGCTTCACGAATTTGCGT \\
GAPDH & F: ACAACTTTGGTATCGTGGAAGG \\
& R: GCCATCACGCCACAGTTTC
\end{tabular}

F, forward primer; R, reverse primer; FZD2, frizzled class receptor 2; MALAT1, metastasis associated lung adenocarcinoma transcript 1; miR, microRNA.

Dual-luciferase reporter assays. For the luciferase reporter assays, HeLa cells $\left(3 \times 10^{4}\right)$ without Cas-II-gly treatment were plated in a 24-well plate and then co-transfected with $400 \mathrm{ng}$ pc3.1-miR-17-5p (miR-17-5p mimics) or pcDNA3.1 empty vector (miR-NC) and $200 \mathrm{ng}$ wild-type (WT) or mutant (mut) luciferase constructs (Sigma-Aldrich; Merck KGaA) using Lipofectamine 2000, according to manufacturer's protocols. HeLa cells were co-transfected with: miR-17-5p NC and MALAT1-WT; miR-17-5p mimics and MALAT1-WT; miR-17-5p NC and MALAT1-mut; miR-17-5p mimics and MALAT1-mut; miR-17-5p NC and FZD2-WT; miR-17-5p mimics and FZD2-WT; miR-17-5p NC and FZD2-mut; or miR-17-5p mimics and FZD2-mut. A Dual-Luciferase Reporter Gene Test kit (Beyotime Institute of Biotechnology, Shanghai, China) was used to detect the luciferase activities in the different groups. The firefly and the Renilla luciferase reagents were added to detect the luciferase activity in each group. Relative luciferase activities were calculated as ratios of firefly to Renilla luciferase activities at $36 \mathrm{~h}$ post-transfection.

Cell viability assays. Cell viability was assessed using an MTT assay (Roche Diagnostics GmbH, Mannheim, Germany). Following treatment with different Cas-II-gly concentrations or transfected with plasmid, according to the aforementioned protocols, HeLa and CaSki cells were plated into a 96-well plate $\left(2 \times 10^{5}\right.$ cells/well) and then incubated for $48 \mathrm{~h}$ at $37^{\circ} \mathrm{C}$. Subsequently, $10 \mu \mathrm{l}$ MTT $(5 \mathrm{mg} / \mathrm{ml})$ was added followed by incubation for $3 \mathrm{~h}$ at $37^{\circ} \mathrm{C}$ in darkness. Following removal of the MTT solution, the precipitated formazan was dissolved by the addition of $100 \mu \mathrm{l}$ dimethyl sulfoxide. After a 10-min incubation at $37^{\circ} \mathrm{C}$ with shaking, the absorbance was quantified with a spectrophotometer at $560 \mathrm{~nm}$ using with a microplate reader (Thermo Fisher Scientific, Inc.).

EdU staining. A Click-iT Plus EdU Alexa Fluor 1594 Imaging kit (Invitrogen; Thermo Fisher Scientific, Inc.) was used to measure cell proliferation, according to the manufacturer's protocol. Transfected HeLa and CaSki cells, according to the aforementioned protocol, were fixed with $50 \mu \mathrm{l}$ cold $4 \%$ formaldehyde for $30 \mathrm{~min}$ at room temperature. DAPI $(1: 2,000)$ was used to stain the cell nuclei for $30 \mathrm{~min}$ at room temperature, and the signal was detected using an Olympus FLUOVIEW FV1000 confocal laser-scanning microscope (Olympus Corporation, Tokyo, Japan) at a magnification of x100.

Cell apoptosis assays. HeLa and CaSki cell apoptosis was measured using fluorescein isothiocyanate (FITC)-conjugated Annexin V and propidium iodide (PI) (BD Pharmingen; Becton-Dickinson and Company, Franklin Lakes, NJ, USA). The cells were washed twice in cold PBS and then resuspended in Annexin V-binding buffer (BD Pharmingen; Becton-Dickinson and Company) at a concentration of $3 \times 10^{6}$ cells $/ \mathrm{ml}$. Additionally, $100 \mu \mathrm{l}$ of the suspension was incubated with $5 \mu \mathrm{l}$ Annexin V-FITC and $5 \mu \mathrm{l} \mathrm{PI}$ in dark at $37^{\circ} \mathrm{C}$ for $15 \mathrm{~min}$. After gentle vortexing, the cells were incubated for $15 \mathrm{~min}$ at room temperature in the dark. Following the addition of $400 \mu \mathrm{l}$ binding buffer (Sigma-Aldrich; Merck KGaA) to each tube, the cells were analyzed using FACSAria ${ }^{\mathrm{TM}}$ III Cell Sorter flow cytometer (BD Biosciences, San Jose, CA, USA) and analyzed using FlowJo V10 software (FlowJo LLC, Ashland, OR, USA).

Statistical analysis. All values were expressed as mean value \pm standard deviation. The data were analyzed using the GraphPad Prism v7.0 software (GraphPad Software, Inc., La Jolla, CA, USA). Unpaired Student's t-test was used to analyze the significance between the measurements of two independent groups and one-way analysis of variance followed by the Tukey post hoc test was used to make comparisons in data sets containing multiple groups. $\mathrm{P}<0.05$ was considered to indicate a statistically significant difference.

\section{Results}

Differentially-expressed mRNAs and lncRNAs following Cas-II-gly treatment. Fig. 1A demonstrates that there were 723 differentially-expressed mRNAs, among which 244 were overexpressed and 479 were suppressed in the Cas-II-gly treatment group. The top 10 upregulated and top 10 downregulated mRNAs are depicted in the heatmap on the left. Compared with the control group, FZD2 was significantly decreased in the Cas-II-gly-treated HeLa cells (Fig. 1A). In reference to lncRNAs, Fig. 1B depicts that only 6 differentially-expressed lncRNAs were identified, including MALAT1. MALAT1 was notably suppressed in comparison with the control group $\left(\mid \log _{2} \mathrm{FCl}>1 ; \mathrm{P}<0.05\right)$.

Functional enrichment of DEGs. The dotplot in Fig. 2A depicts the distribution of the $30 \mathrm{GO}$ biological process terms (15 were activated and 15 were suppressed). The 'cellular response to heat' term was activated in Cas-II-gly-treated HeLa cells, indicating that Cas-II-gly may disrupt proper protein folding as a result of nonspecific aggregation (39). The 'cell cycle' term, which involves cell division and DNA replication, was suppressed in the treatment group. The horizontal axis represents the gene ratio, which was the result of the gene count divided by the set size. In the enrichment map depicted in Fig. 2B, larger sets of the enrichment results were combined 


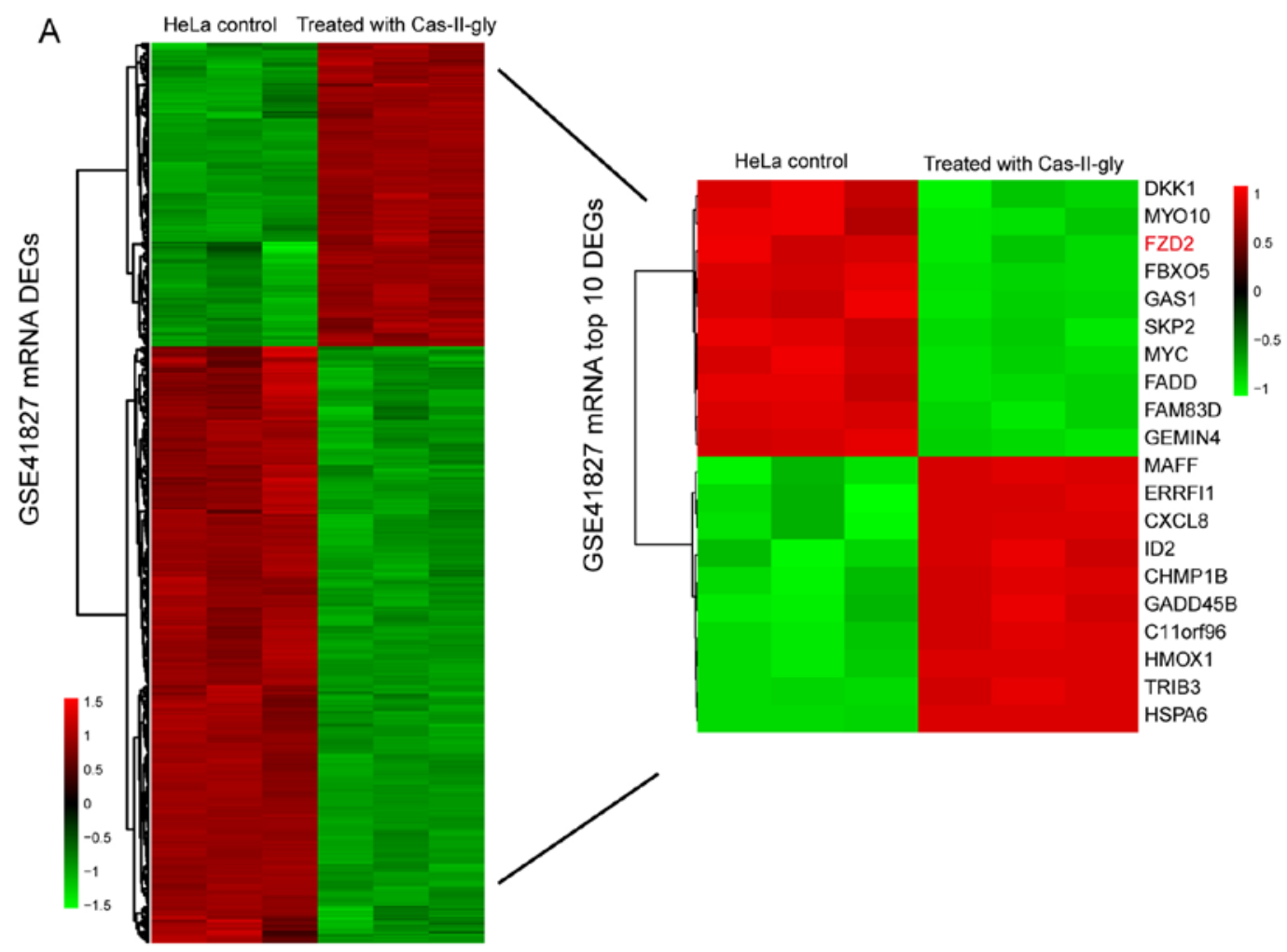

B

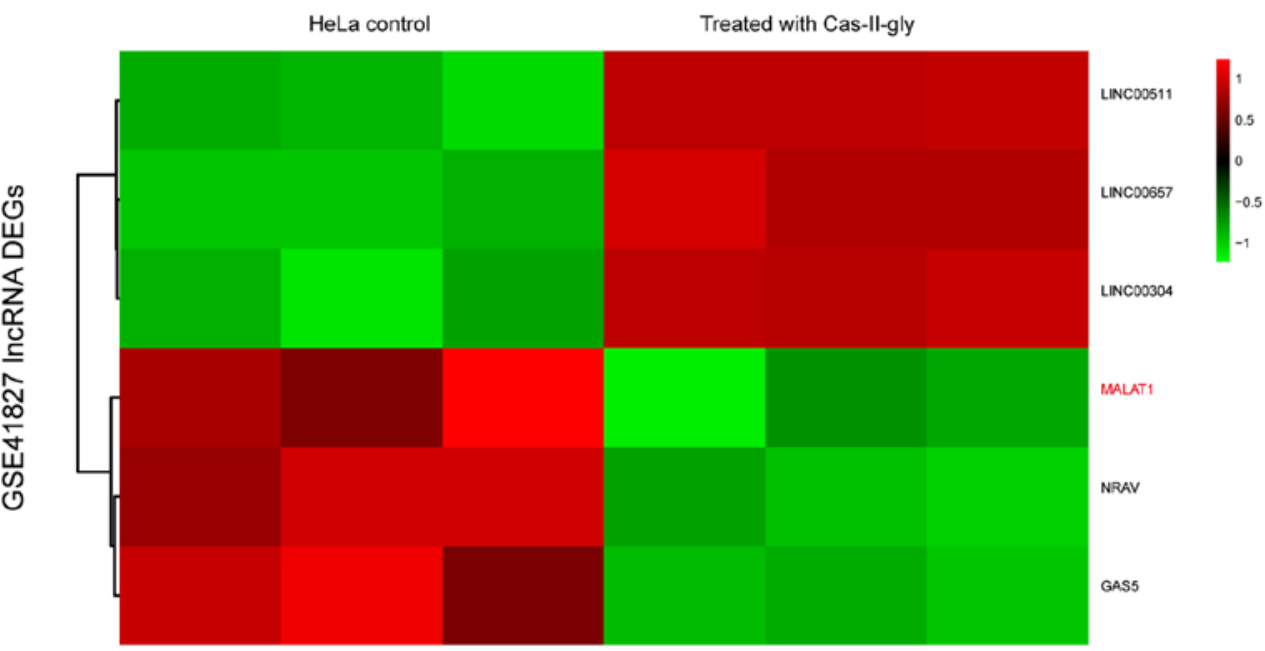

Figure 1. DEGs in cervical cells in the treatment and control groups. (A) Left: Total differentially-expressed mRNAs in the Cas-II-gly-treated and control groups, according to GSE41827. Right: The top 10 upregulated and downregulated mRNAs in the Cas-II-gly-treated and control groups, according to GSE41827. FZD2 was suppressed in the Cas-II-gly group. (B) Total differentially-expressed lncRNAs in the Cas-II-gly-treated and control groups, according to GSE41827. MALAT1 was suppressed in the Cas-II-gly-treated group. Cas-II-gly, Casiopeina II-gly; FZD2, frizzled class receptor 2; lncRNA, long non-coding RNA; MALAT1, metastasis associated lung adenocarcinoma transcript 1; DEGs, differentially-expressed genes.

into a network to gather highly similar terms into clusters to highlight overall trends (40). The 'cellular response to unfolded protein', 'response to unfolded protein' and 'cellular response to topologically incorrect protein' terms had intersecting gene sets with each other. The ridgeplot in Fig. $2 \mathrm{C}$ depicts the exact gene set expression of these biological process terms and the enrichment results of the biological process terms. If the peak values are $<0$, then the terms were suppressed, and the terms with peak values $>0$ were activated. For instance, the 'apoptotic mitochondrial changes' term, which reflects the cell metabolism level, was activated in the treatment group. In cnetplot in Fig. 2D depicts the association between the 5 biological process-enriched terms and the DEGs, including the "cell division', 'DNA package', 'apoptotic mitochondrial changes', 'regulation of transcription from RNA polymerase II promoter in response to stress' and 'regulation of DNA-templated transcription in response to stress' terms.

Wnt signaling pathway is suppressed. Fig. 3A depicts the distribution of 15 activated and 15 suppressed signaling pathways. The Wnt signaling pathway was notably suppressed in Cas-II-gly-treated HeLa cells. Furthermore, the exact gene 
A

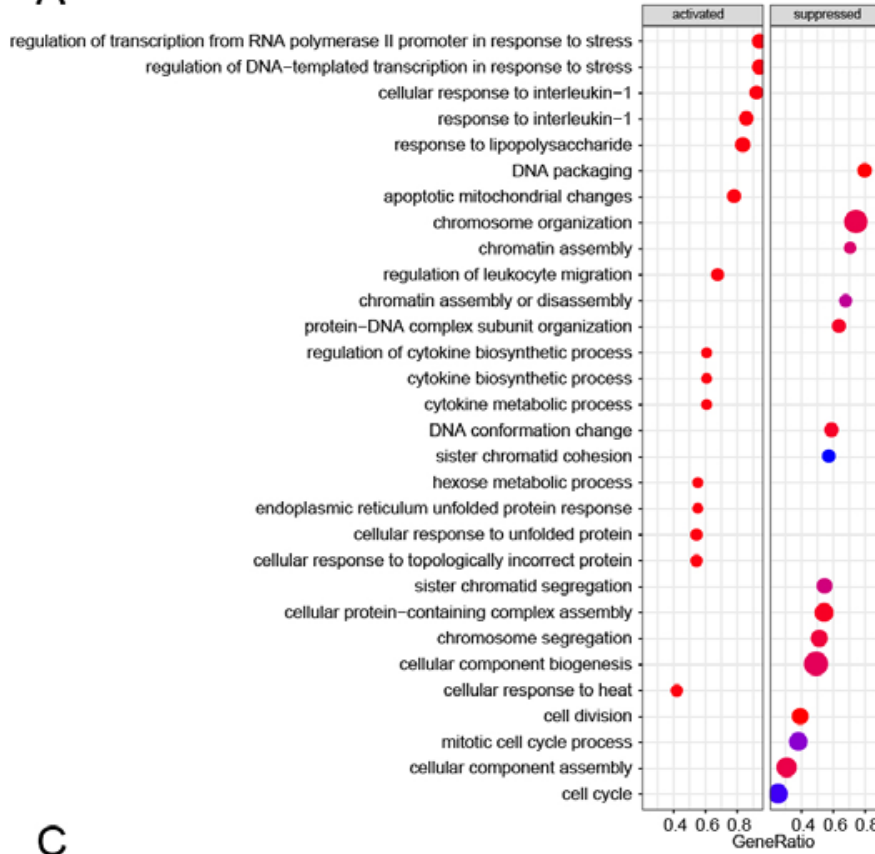

C

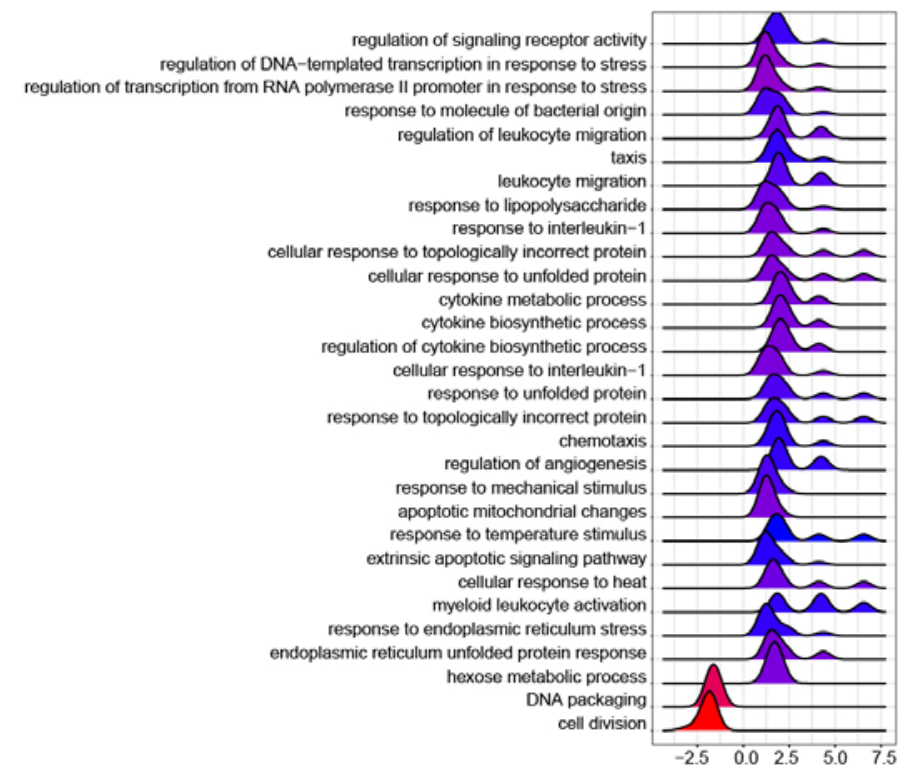

B

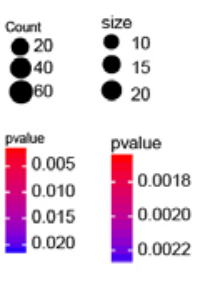

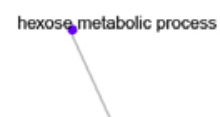

regulation of cyokine biosynthetic process cellular response to topologically incorrect protein cytokie biosynthetic process endoplasmic reficulum unfolded protein response

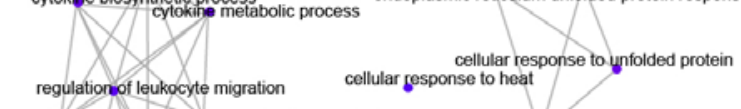

leukecyte migration cellular response to interleukin-1 response to molecule of bacterial origin response to lipopolyseccharide rution of DNA-templated transcription in response to stress regulation of transcription from RNA polymerase 11 promoter in response to stress

D

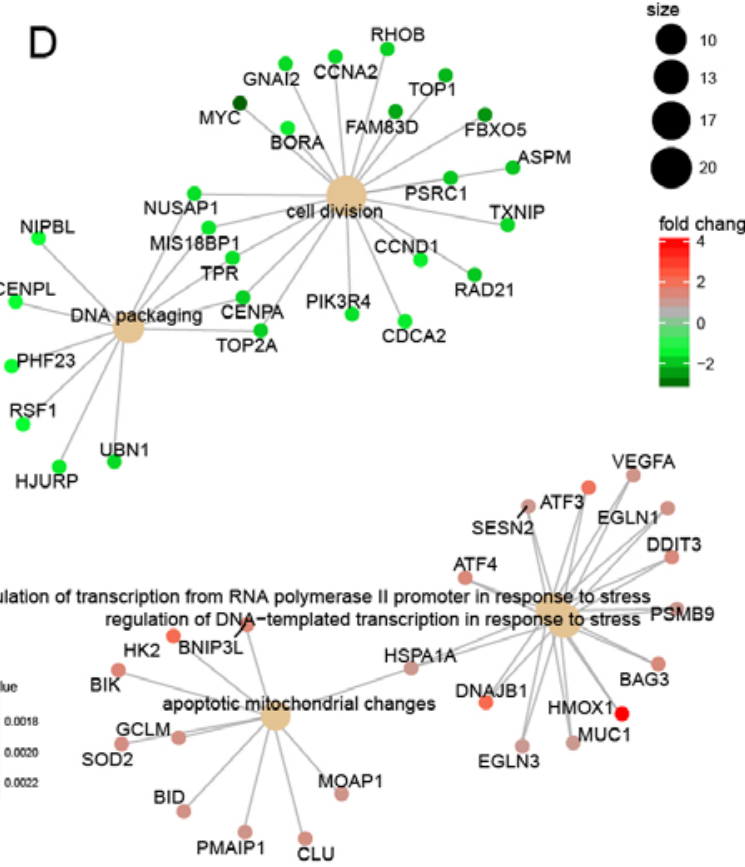

Figure 2. Functional enrichment analysis visualization. (A) The dotplot depicts the activity of biological processes terms. (B) The enrichment map depicts the association between the enriched biological processes terms. (C) The ridgeplot depicts the exact gene set expression and enrichment results of the biological processes. (D) The cnetplot depicts the detected enriched five biological processes and their associated differentially-expressed genes.

set expression and enrichment results are depicted in the ridgeplot in Fig. 3B. The Wnt signaling pathway was significantly suppressed, as its peak was $<0$. The running enrichment score of the Wnt signaling pathway is depicted in Fig. 3C, which indicates the suppression of the Wnt signaling pathway. Based on these combined bioinformatics results, it was concluded that the Wnt signaling pathway may participate in the Cas-II-gly dependent effects on HeLa cells. The correlation analysis Fig. 3D depicts the association between the DEGs in the Wnt signaling pathway and the differentially-expressed lncRNAs. $F Z D 2$ was the upstream core gene in the Wnt signaling pathway, which was downregulated in the treatment group. The MALAT1 IncRNA had positive correlation with FZD2.
Targeting association between miR-17-5p and MALATI/FZD2 . Fig. 4A depicts that there were 69 miRNAs targeting FZD2 in the TargetScan database and 113 miRNAs targeting the MALAT1 lncRNA in the starBase database. A total of 10 miRNAs were common between these two databases and hsa-miR-17-5p was selected as the target miRNA. Fig. 4B depicts the target sites that hsa-miR-17-5p could bind in the 3'-untranslated regions of MALAT1 and FZD2 determined in the starBase database. The target sites were further verified using a luciferase reporter assay. As depicted in Fig. 4C and D, miR-17-5p overexpression resulted in a significant decrease in luciferase activity in MALAT1-WT without changing the luciferase activity of MALAT1-mut in HeLa cells, which 
A

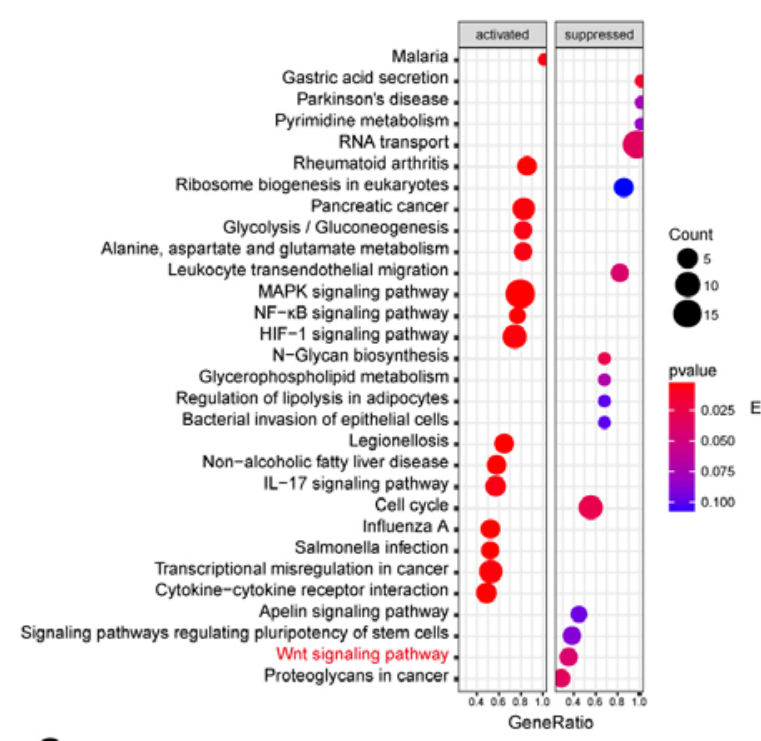

C

KEGG_WNT_SIGNALING_PATHWAY

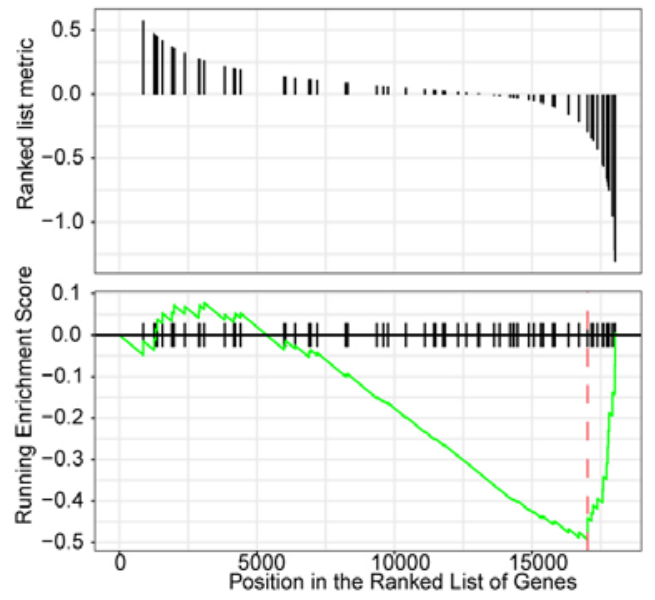

B

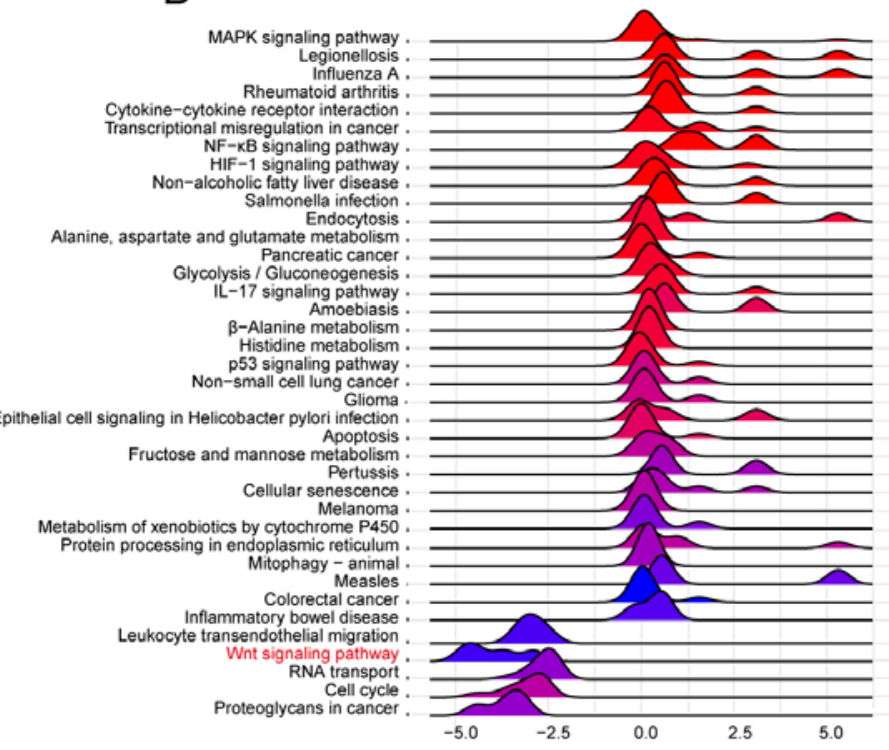

$\mathrm{D}$

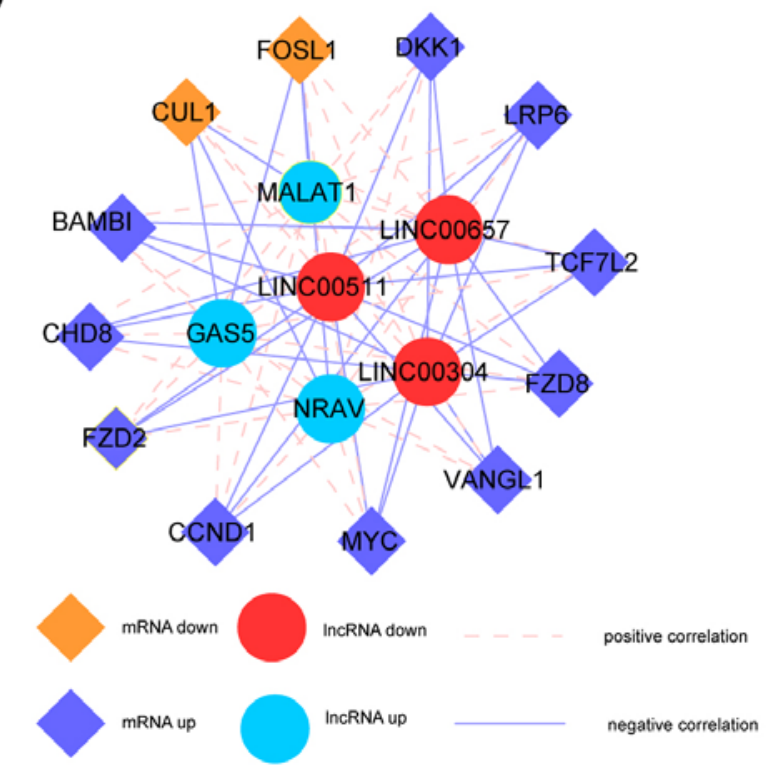

Figure 3. Pathway enrichment visualization. (A) The dotplot depicts the activity of the enriched signaling pathways. The Wnt signaling pathway was suppressed in HeLa cells treated with Casiopeina II-gly. (B) The ridgeplot depicts the exact gene set expression and enrichment results of the signaling pathways. The peak for the Wnt signaling pathway was $<0$, so it was suppressed. (C) In the GSEAplot, the running score of the Wnt signaling pathway was $<0$, indicating that this pathway was suppressed. (D) The correlations between the differentially-expressed genes in the Wnt signaling pathway and the differentially-expressed lncRNAs. KEGG, Kyoto Encyclopedia of Genes and Genomes; lncRNAs, long non-coding RNAs.

confirmed that miR-17-5p binds to MALAT1 via the target sites. A similar result was obtained in the verification of the targeting association between miR-17-5p and FZD2, as depicted in Fig. 4E and F.

Cas-II-gly regulates the expression of MALAT1, miR-17-5p and FZD2. To further investigate whether Cas-II-gly can regulate the MALAT1-miR-17-5p-FZD2 axis, the expression profiles of MALAT1, miR-17-5p and FZD2 was measured in HeLa and CaSki cells treated with increasing doses of Cas-II-gly. Fig. 5A and B depicts that the expression of MALAT1 was significantly suppressed with increasing Cas-II-gly concentrations. The opposite result is depicted in Fig. 5C and D, as the miR-17-5p expression significantly increased with increasing Cas-II-gly concentrations. The western blot results depicted in Fig. 5E-H indicate that FZD2 was also significantly inhibited by Cas-II-gly. Subsequently, to determine the optimum Cas-II-gly concentration, MTT assays were performed to assess the influence of Cas-II-gly on the proliferation of HeLa, CaSki and HcerEpic cells. The results in Fig. 5I-K demonstrate that $10 \mathrm{mM}$ Cas-II-gly could inhibit the proliferation of HeLa and CaSki cells, with no significant killing effect on the HcerEpic cells; therefore, $10 \mathrm{mM}$ Cas-II-gly was selected for the subsequent experiments.

MALAT1 regulates FZD2 expression by targeting miR-17-5p. To modulate the expression levels of MALAT1, pcDNA 
A

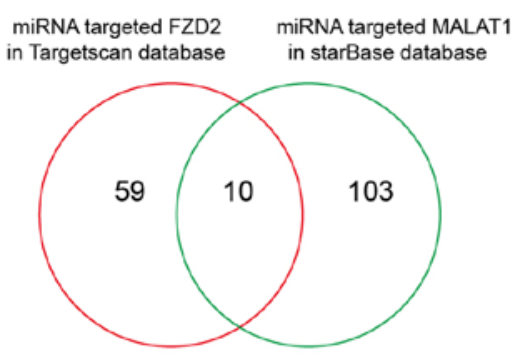

C

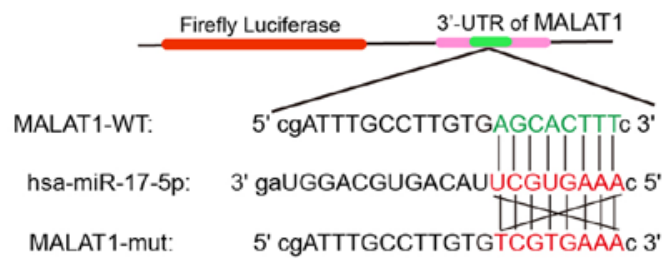

E

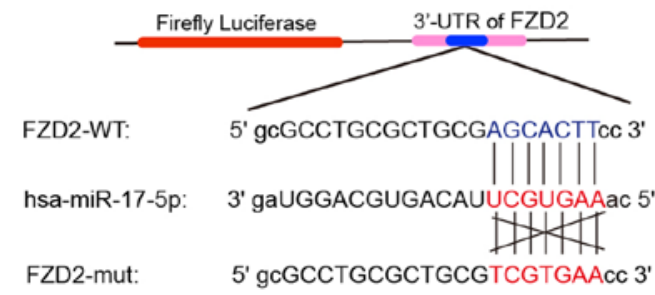

B

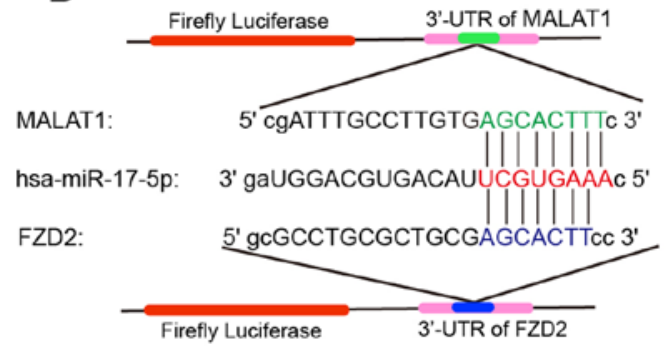

D

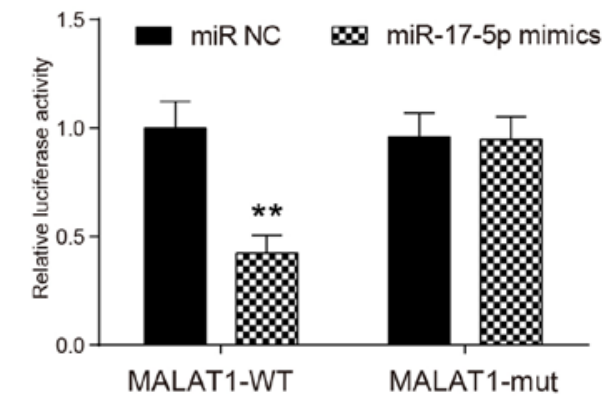

$\mathrm{F}$

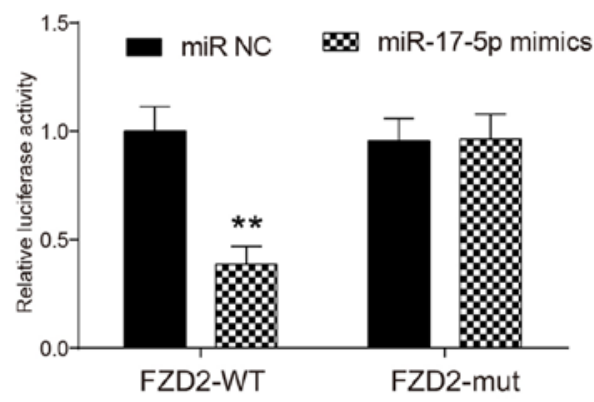

Figure 4. The targeting association between miR-17-5p and MALAT1/FZD2. (A) The intersection of the TargetScan and starBase results, and miR-17-5p served as the targeting miRNA. (B) The binding sites for miR-17-5p, MALAT1 and FZD2. (C) The sequences of miR-17-5p and MALAT1-WT/MALAT1-mut. (D) A dual-luciferase reporter assay verified the target association between miR-17-5p and MALAT1. (E) The sequences of miR-17-5p and FZD2-WT/FZD2-mut. (F) A dual-luciferase reporter assay verified the targeting association between miR-17-5p and FZD2. ${ }^{* *} \mathrm{P}<0.01 \mathrm{vs}$. the NC group. miR, microRNA; FZD2, frizzled class receptor 2; MALAT1, metastasis associated lung adenocarcinoma transcript 1; UTR, untranslated region; NC, negative control; WT, wild-type; mut, mutation.

3.1-MALAT1 and si-MALAT1 were designed to overexpress or knockdown MALAT1 expression, respectively. As depicted in Fig. 6A-D, the expression levels of MALAT1 and FZD2 were significantly increased by pcDNA 3.1-MALAT1 and decreased by si-MALAT1, while the opposite effect was observed for miR-17-5p expression. Similarly, miR-17-5p mimics and the inhibitor were designed to modulate the expression levels of miR-17-5p. The results in Fig. 6E-H indicate that the miR-17-5p mimics could significantly increase miR-17-5p expression and significantly suppress $F Z D 2$ expression, while miR-17-5p inhibitor had the opposite effects. However, the miR-17-5p mimics and the inhibitor did not significantly affect MALAT1 expression. The results of the western blot analysis depicted in Fig. $6 \mathrm{I}$ and $\mathrm{J}$ demonstrated that pcDNA 3.1-FZD2 could significantly enhance $F Z D 2$ expression and si-FZD2 could significantly reduce it.

Cas-II-gly affects CC cells via the MALATI/miR-17-5p/FZD2 axis. Toinvestigate the effects of these factors on the proliferation and apoptosis of HeLa and CaSki cells, the experiments were divided into 8 groups: NC; $10 \mathrm{mM}$ Cas-II-gly; si-MALAT1; mimics; si-FZD2; MALAT1 + mimics; inhibitor + si-FZD2; and si-MALAT1 + FZD2. Fig. 7A-D depicts the EdU staining in HeLa and CaSki cells, and the results indicated that $10 \mathrm{mM}$ Cas-II-gly, si-MALAT1, mimics and si-FZD2 could significantly reduce the proliferation of HeLa and CaSki cells, while MALAT1 + mimics, inhibitor + si-FZD2 and si-MALAT1 $+F Z D 2$ did not cause any significant changes in cell proliferation. The effects on apoptosis in HeLa and CaSki cells are depicted in Fig. 7E-H. Opposite results were from those of the EdU staining were obtained, with $10 \mathrm{mM}$ Cas-II-gly, si-MALAT1, mimics and si-FZD2 significantly increasing the apoptosis rate of $\mathrm{HeLa}$ and CaSki cells, while MALAT1 + mimics, inhibitor + si-FZD2 and si-MALAT1 + FZD2 had no significant effects. In conclusion, all these data indicate that Cas-II-gly can affect the proliferation and apoptosis of CC cells via the MALAT1/miR-17-5p/FZD2 axis. 

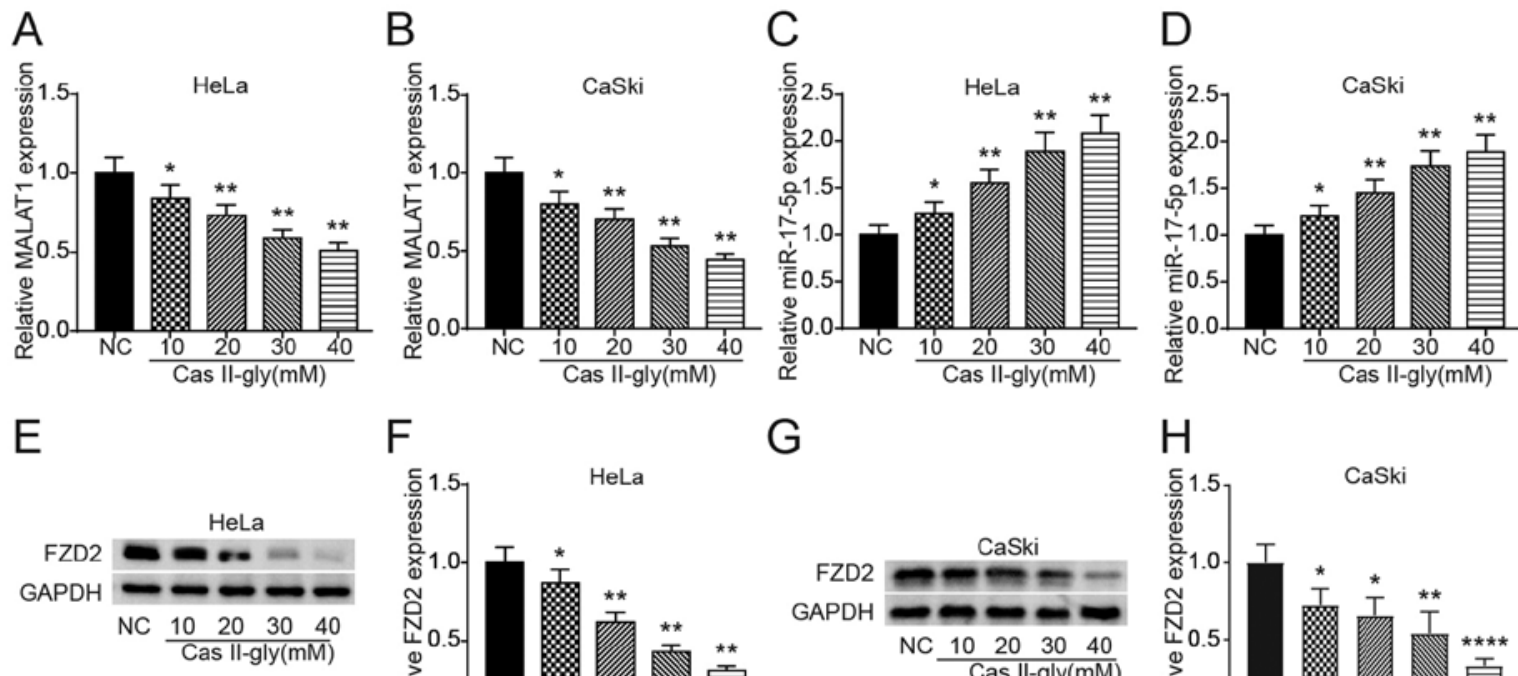

\section{$\mathrm{F}$}

G
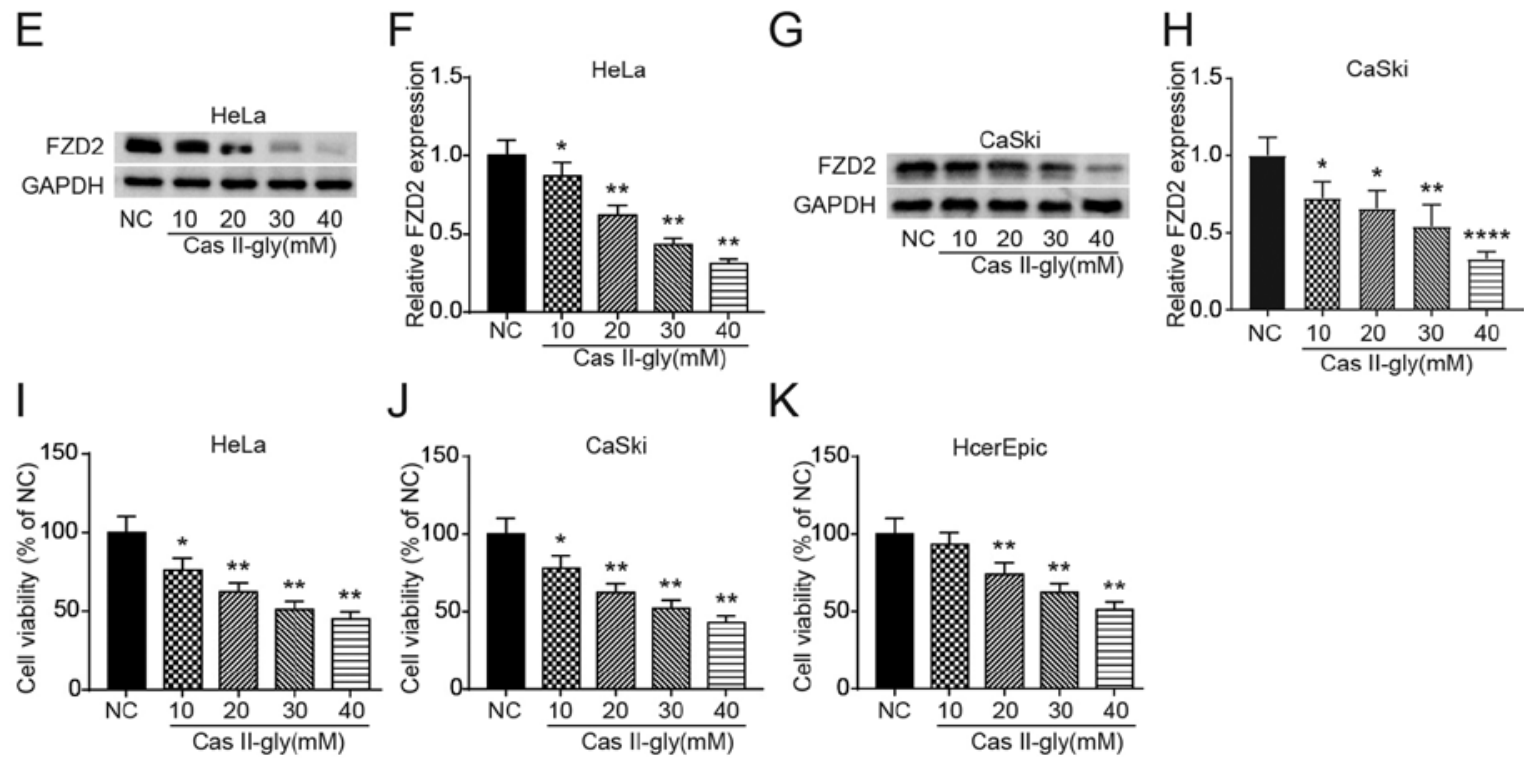

Figure 5. The effect of Cas-II-gly on MALAT1, miR-17-5p and FZD2. The experiments were divided into 5 groups: NC; $10 \mathrm{mM}$ Cas-II-gly; $20 \mathrm{mM}$ Cas-II-gly; $30 \mathrm{mM}$ Cas-II-gly; and $40 \mathrm{mM}$ Cas-II-gly. Reverse transcription-quantitative polymerase chain reaction was used to measure the expression levels of MALAT1 in (A) HeLa and (B) CaSki cells, and miR-17-5p in (C) HeLa and (D) CaSki cells. (E) Western blotting images and (F) densitometric quantification of FZD2 in HeLa cells. (G) Western blotting images and $(\mathrm{H})$ densitometric quantification of FZD2 in CaSki cells. An MTT assay was used to detect the proliferation of (I) HeLa, (J) CaSki and (K) HcerEpic cells. ${ }^{*} \mathrm{P}<0.05$ and ${ }^{* *} \mathrm{P}<0.01$ vs. the NC group. Cas-II-gly, Casiopeina II-gly; FZD2, frizzled class receptor 2; MALAT1, metastasis associated lung adenocarcinoma transcript 1; NC, negative control.

Cas-II-gly regulates the Wnt signaling pathway in CC cells via the MALAT1/miR-17-5p/FZD2 axis. At the conclusion of the present experiments, western blot analysis was performed to assess the levels of Wnt signaling pathway-associated proteins (Dvl, GSK-3 $\beta$ and $\beta$-catenin) in $\mathrm{HeLa}$ and $\mathrm{CaSki}$ cells. As the results in Fig. 8A-D depict, $10 \mathrm{mM}$ Cas-II-gly, si-MALAT1, mimics and si-FZD2 could increase the expression of Dvl, $\beta$-catenin and GSK-3 $\beta$ in HeLa and CaSki cells, and no significant effects on these proteins were observed for MALAT1 + mimics, inhibitor + si-FZD2 and si-MALAT1 + FZD2. Additionally, all these data demonstrate that Cas-II-gly can regulate the Wnt signaling pathway via the MALAT1/miR-17-5p/FZD2 axis in HeLa and CaSki cells.

\section{Discussion}

In the present study, it was determined that Cas-II-gly altered the expression levels of the IncRNA MALAT1, the mRNA FZD2 and suppressed the Wnt signaling pathway in HeLa and CaSki cells. In vitro experiments were conducted to further investigate the effects of Cas-II-gly on gene expression and cell functions, and the results indicated that Cas-II-gly suppressed the progression of CC cells by suppressing the MALAT1/miR-17-5p/FZD2/Wnt signaling pathway axis.
Cas-II-gly has been reported to be beneficial in the treatment of cancer with limited side effects (8). In the present study, the underlying mechanism, which is not yet fully understood, was investigated. A number of studies have revealed outcomes associated with Cas-II-gly treatment. In cervix-uterine and neuroblastoma tumors, Cas-II-gly may result in mitochondrial apoptosis (7). More specifically, it was reported that it inhibited the state 3 rates and uncoupled respiration in mitochondria (41), and that it induced cytotoxicity associated with oxidative damage (8). Correia et al (42) demonstrated that Cas-II-gly may be transported by human serum albumin, as low Cas-II-gly concentrations are expected to be found in blood, which may serve as a selective tumor target. Another study revealed that Cas-II-gly may activate the MAPK signaling pathway (7), which is involved in cellular programs, including proliferation, differentiation, development, transformation and apoptosis (43). Cas-II-gly had the same effects on other tumor types. For instance, in glioma C6 cells, Cas-II-gly inhibited cell proliferation, increased reactive oxygen species levels and contributed to apoptosis (44). In CH1 human ovarian carcinoma cells, Cas-II-gly may induce apoptosis without exhibiting DNA oligonucleosomal fragments against CC cells (45). A slight decrease in the proliferation of normal cervical epithelial cells was observed following treatment with $40 \mathrm{mM}$ Cas-II-gly. 
A

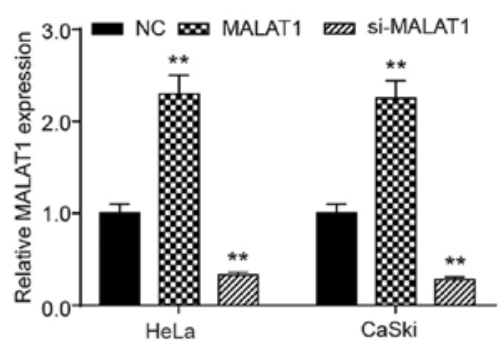

C

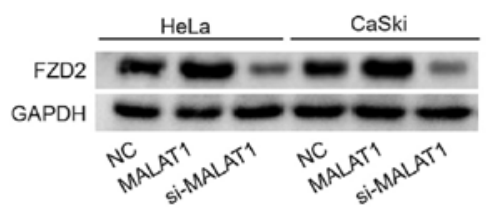

E

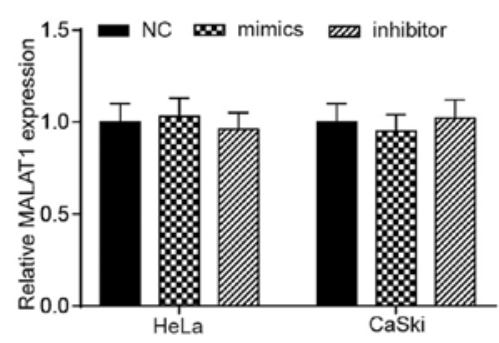

G

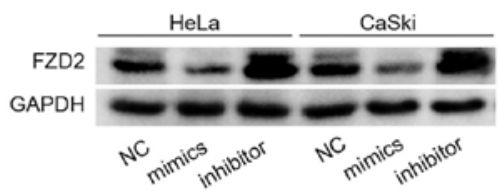

I

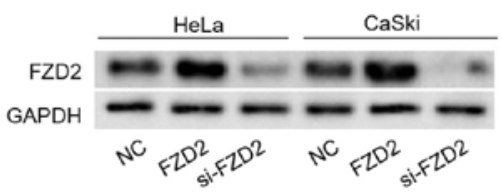

B

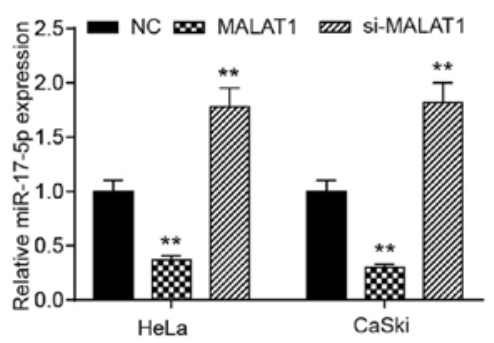

D

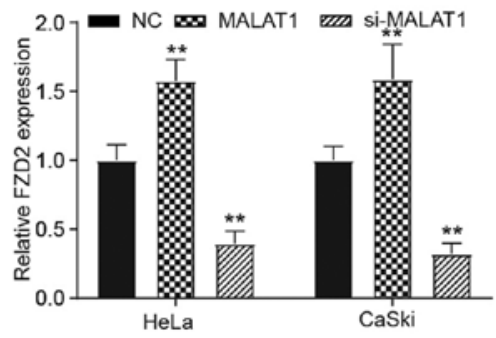

F

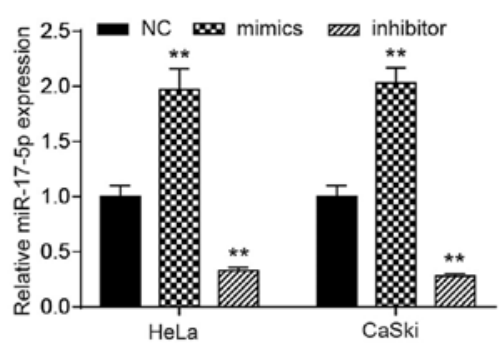

$\mathrm{H}$

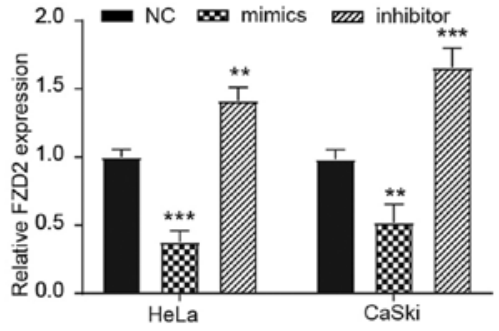

J

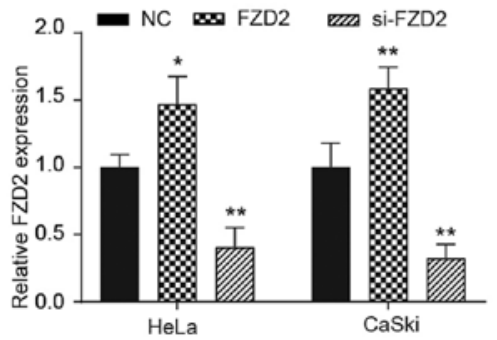

Figure 6. The effects of MALAT1 on miR-17-5p and FZD2. The effects of si-MALAT1 and pcDNA 3.1-MALAT1 on (A) MALAT1 and (B) miR-17-5p expression were confirmed by RT-qPCR. (C) Western blotting images and (D) densitometric quantification of FZD2 in HeLa and CaSki cells. (E) The effects of the miR-17-5p mimics and inhibitor on (E) MALAT1 and (F) miR-17-5p expression were confirmed by RT-qPCR. (G) Western blotting images and $(\mathrm{H})$ densitometric quantification of FZD2 in HeLa and CaSki cells following miR-17-5p mimics and inhibitor incubation. (I) Western blotting images and (J) densitometric quantification of FZD2 in HeLa and CaSki cells following FZD2 overexpression plasmid and si-FZD2 incubation. ${ }^{* *} \mathrm{P}<0.01$ vs. the NC group. RT-qPCR, reverse transcription-quantitative polymerase chain reaction; NC, negative control; si, small interfering; FZD2, frizzled class receptor 2; MALAT1, metastasis associated lung adenocarcinoma transcript 1; miR, microRNA.

Cas-II-gly is a copper-based drug with anticancer activity (8). While this compound has some toxicity (46), it may also have a slight side effect on normal cervical epithelial cells, as indicated by the results of the present experiments.

In the present study, MALAT1 was downregulated in CC cells following Cas-II-gly treatment, indicating that MALAT1 may be a cancer-promoting 1ncRNA that could contribute to tumor development. Xia et al (47) supported this result using metformin. During the process of CC metastasis, miR-142-3p is significantly upregulated, whereas the lncRNA MALAT1 and high mobility group AT-hook 2 were suppressed by metformin (47). Furthermore, 
A NC

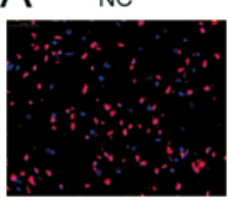

si-FZD2

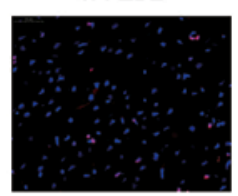

C NC

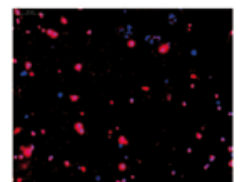

si-FZD2

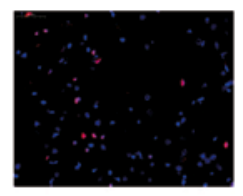

E NC

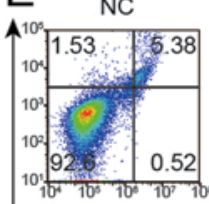

至 si-FZD2

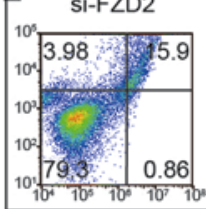

10mM Cas II-gly

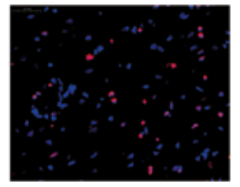

MALAT1+mimics

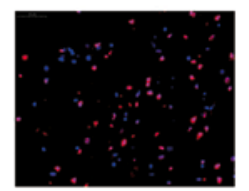

$10 \mathrm{mM}$ Cas II-gly

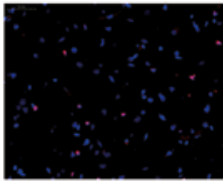

MALAT1+mimics

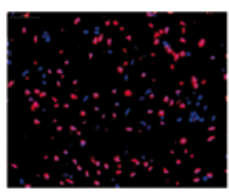

si-MALAT1

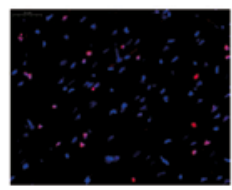

inhibitor+si-FZD2

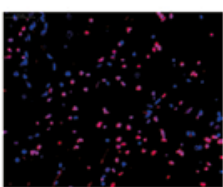

Si-MALAT1

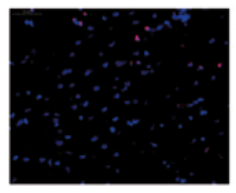

inhibitor+si-FZD2

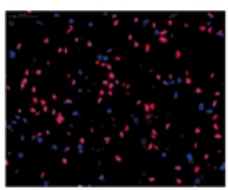

mimics

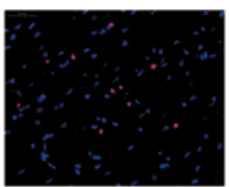

si-MALAT1+FZD2

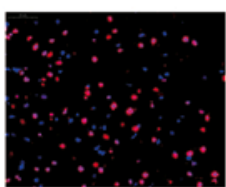

mimics
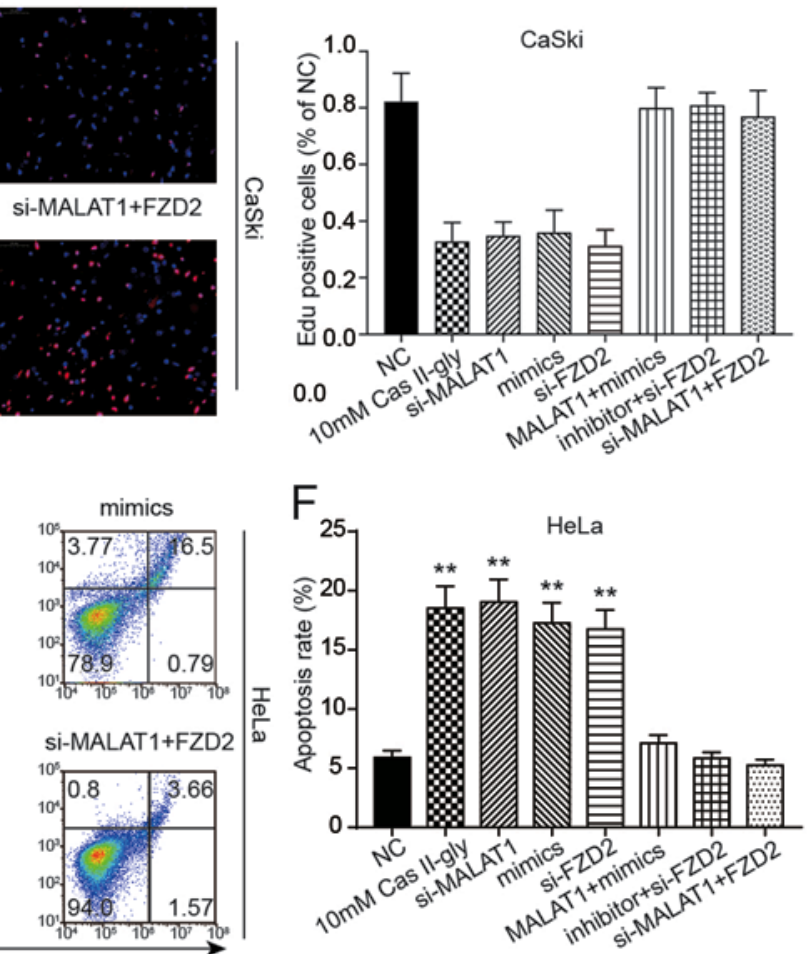

$\mathrm{H}$

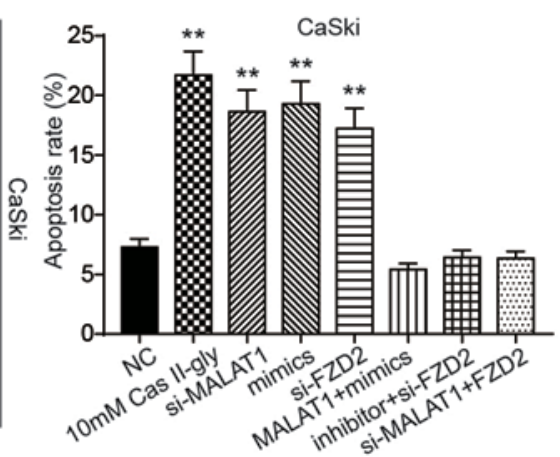

Figure 7. The effects of Cas-II-gly on the proliferation and apoptosis of CC cells. (A) EdU staining images and (B) quantification of proliferating HeLa cells. (C) EdU staining images and (D) quantification of proliferating HeLa cells. (E) Flow cytometry images and (F) quantification of apoptotic HeLa cells. (G) Flow cytometry images and (H) quantification of apoptotic HeLa cells. ${ }^{* *} \mathrm{P}<0.01$ vs. the NC group. Cas-II-gly, Casiopeina II-gly; FZD2, frizzled class receptor 2; MALAT1, metastasis associated lung adenocarcinoma transcript 1; NC, negative control; si, small interfering; FITC, fluorescein isothiocyanate; PI, propidium iodide.

MALAT1 and polypyrimidine tract binding protein 2 were overexpressed, and both are associated with invasion and metastasis in colorectal cancer (48). Besides, they may also be oncogenic through other processes. Some results indicated that MALAT1 promotes proliferation and metastasis in osteosarcoma by activating the PI3K/Akt pathway (49) 
A

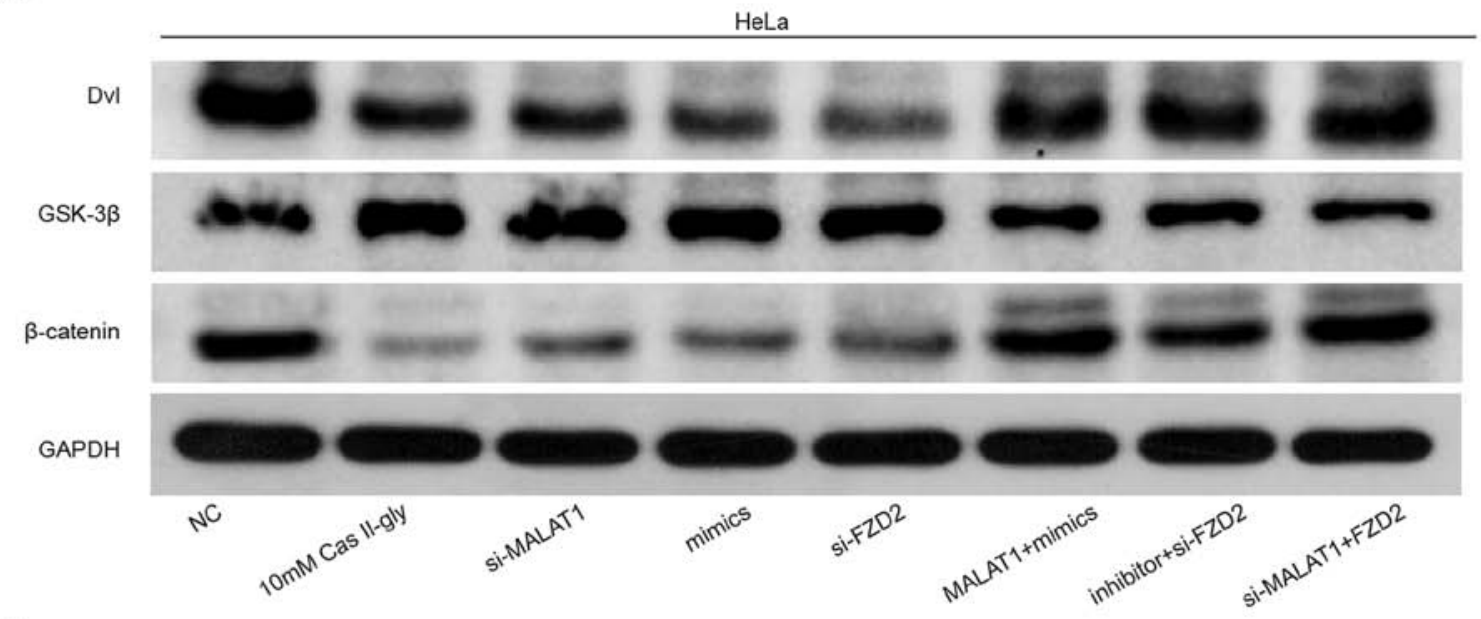

B

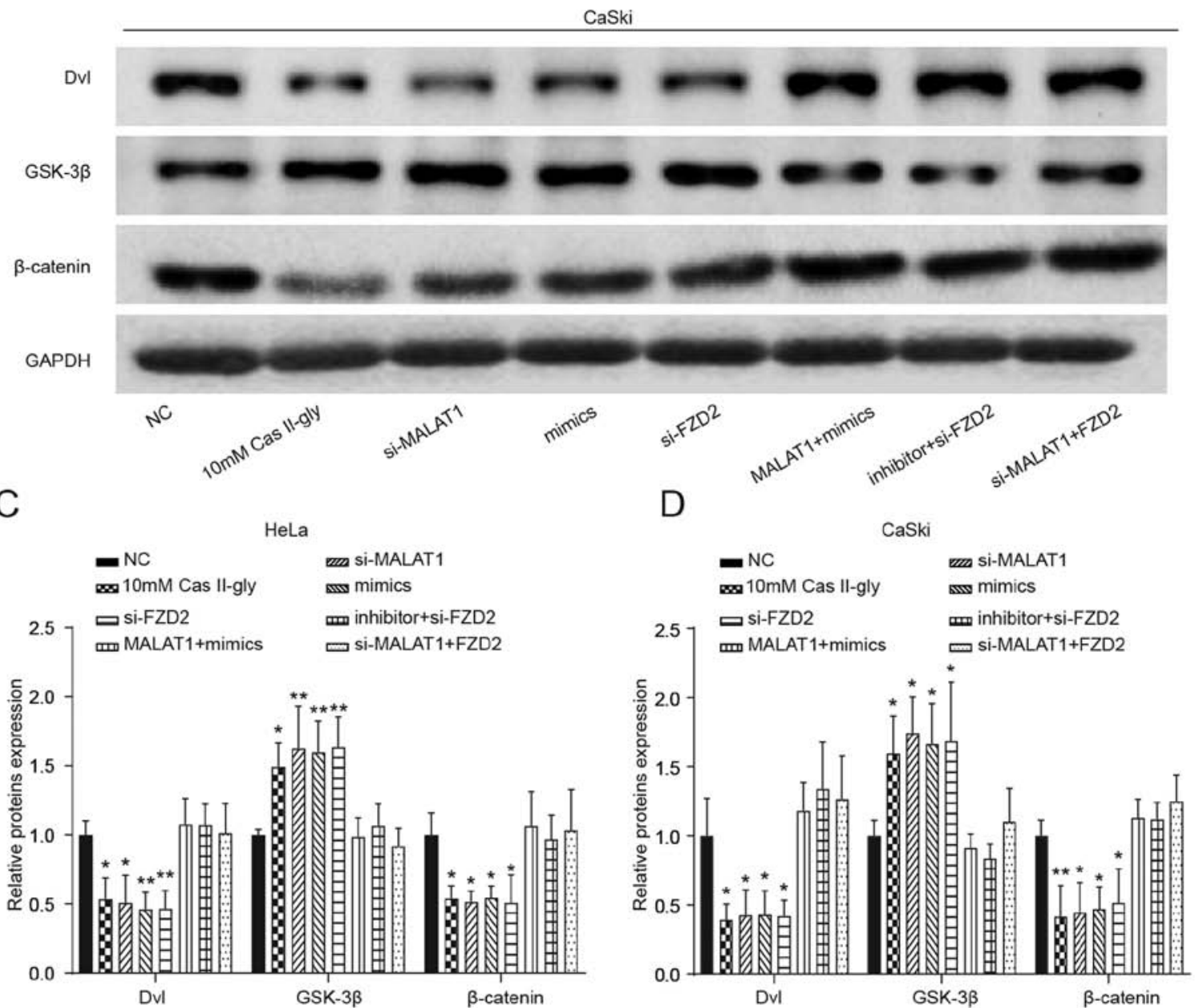

Figure 8. The effects of Cas-II-gly on the Wnt signaling pathway in cervical cancer cells. (A-D) Western blotting was used to measure the levels of Wnt signaling pathway-associated proteins (Dvl, GSK-3 $\beta$ and $\beta$-catenin) in the NC, $10 \mathrm{mM}$ Cas-II-gly, si-MALAT1, mimics, si-FZD2, MALAT1 + mimics, inhibitor + si-FZD2 and si-MALAT1 + FZD2 groups. * P $<0.01$ vs. the NC group. Cas-II-gly, Casiopeina II-gly; FZD2, frizzled class receptor 2; MALAT1, metastasis associated lung adenocarcinoma transcript 1; NC, negative control; si, small interfering; Dvl, disheveled segment polarity protein; GSK-3 $\beta$, glycogen synthase kinase-3 $\beta$.

and by sponging miR-200s in clear cell renal carcinoma (50). Knockdown of MALAT1 expression decreases renal cancer cell proliferation, migration, and invasion (51). All of these data indicated that Cas-II-gly may suppress CC cells by regulating MALAT1; therefore, MALAT1 was selected for the present study.
FZD2, which acts as a Wnt receptor, had reduced expression in HeLa and CaSki cells following Cas-II-gly treatment, indicating its cancer-promoting function in CC. FZD2 belongs to the $\mathrm{Fz}$ gene family, which are well documented to be involved in carcinogenesis. For instance, Li et al (52) determined that receptor tyrosine kinase like orphan receptor 2 
modulates canonical Wnt signaling in lung epithelial cells through FZD2. They demonstrated that FZD2 overexpression in HEC-1B and Ishikawa cells promoted their migration and that it induced an epithelial-mesenchymal transition phenotype in endometrial cancer (53). Another study demonstrated that overexpression of $F Z D 2$ promoted tongue squamous cell migration and invasion, while RNA interference-mediated $F Z D 2$ knockdown had opposite effects on tongue squamous cell migration and invasion (54). However, FZD2 expression was reduced in metastatic salivary adenoid cystic carcinoma tissue, compared with tissue without metastasis (55). A possible reason for this difference may be that the metastatic tumor has more complex behaviors, compared with the original tumor.

Garzon et al (56) reported that $\mathrm{miR}-17-5 \mathrm{p}$ acts as a tumor suppressor in prostate, cervical and breast cancer. In the present study, it served as an inhibitor of FZD2 and was demonstrated to be a target of MALAT1. Wei et al (57) demonstrated that miR-17-5p functions as a tumor suppressor in CC cells by targeting TP53INP1. It was reported that miR-17-5p expression was downregulated, while $\alpha-1,2$ fucosyltransferase 2 expression was increased during osteoarthritis progression (58). Another study demonstrated that miR-17 downregulation promoted B cell CLL/lymphoma 11B overexpression in $\mathrm{T}$ cells and promoted the development of acute lymphoblastic leukemia (59). Furthermore, Li et al (60) revealed that miR-17-5p and miR-20a alleviate the suppressive function of myeloid-derived suppressor cells by modulating signal transducer and activator of transcription 3 expression in acute myeloid leukemia. Furthermore, miR-17-5p was demonstrated to sensitize MCF-7 cells (a breast cancer cell line) to paclitaxel-induced apoptosis (15), and these data indicated that miR-17-5p may be a potential target miRNA of MALAT1 and that it could be involved in the antitumor mechanism of Cas-II-gly in CC; therefore, miR-17-5p was selected for further study.

In the present study, the involvement of the MALAT1-miR-17-5p-FZD2 axis during the treatment of $\mathrm{CC}$ cells with Cas-II-gly was revealed. MALAT1 acted as a competing endogenous RNA for miR-126-5p to modulate FZD2 expression, resulting in the activation of the Wnt signaling pathway. Liu et al (61) indicated that MALAT1 could promote high risk human papillomavirus (+) CC cell growth and invasion, at least partially through the MALAT1-miR-124-RBG2 axis. As previously described, MALAT1 may not only function as an oncogene but could also serve as a drug target (47). Furthermore, MALAT1 and miR-17-5p were demonstrated to be associated with chemoresistance $(15,62,63)$. Consistent with other studies, the present data demonstrated that Cas-II-gly targets the MALAT1-miR-17-5p-FZD2 axis and that it inactivates the Wnt signaling pathway, thus resulting in cell apoptosis.

In the present study, it was determined that the Wnt pathway was suppressed in the Cas-II-gly treatment group, resulting in apoptosis. Additionally, other studies supported this result. For example, Pećina-Slaus et al (64) reported that the Wnt pathway regulates apoptosis. More specifically, Wnt-1 functions as an antiapoptotic signal, and that it inhibits apoptosis by activating $\beta$-catenin/T cell factor-mediated transcription (65). Similarly, You et al (66) determined that a $\beta$-catenin-independent non-canonical pathway, including the Wnt/JNK pathway, may serve a role in the Wnt-1 signaling-dependent inhibition of apoptosis. In other cancer types, it was also demonstrated that the Wnt pathway was involved in apoptosis. For instance, Wnt signaling regulates the early and late stages of apoptosis during development and cellular injury in neurons, endothelial cells, vascular smooth muscle cells and cardiomyocytes (67). Additionally, in non-small cell lung cancer, the Wnt pathway was activated by disheveled overexpression, which contributed to tumor development (68).

In conclusion, it was demonstrated that Cas-II-gly acts on MALAT1 by targeting miR-17-5p to inhibit FZD2 expression via inactivation of the Wnt signaling pathway, thus inhibiting cell proliferation and promoting apoptosis in HeLa and CaSki CC cells.

\section{Acknowledgements}

Not applicable.

\section{Funding}

The present study was supported by grants from the National Natural Science Foundation of China (grant nos. 81771531, 81571395, 81671408 and 81701634).

\section{Availability of data and materials}

All data generated or analyzed during this study are included in this published article.

\section{Authors' contributions}

YX and RO conducted critical revision of the manuscript. YX, QZ and FL provided substantial contribution to the conception and design of the work, and manuscript drafting. LZ, FH and LZ conducted acquisition, analysis and interpretation of the data. YX and RO revised the manuscript critically and provided final approval of the version to be published. All authors have read and approved the final article.

\section{Ethics approval and consent to participate}

The present study was authorized by ethics committee of The First Affiliated Hospital of Wenzhou Medical University.

\section{Patient consent for publication}

Not applicable.

\section{Competing interests}

The authors declare that they have no competing interests.

\section{References}

1. Siegel RL, Miller KD and Jemal A: Cancer statistics, 2018. CA Cancer J Clin 68: 7-30, 2018.

2. Tewari KS and Monk BJ: New strategies in advanced cervical cancer: From angiogenesis blockade to immunotherapy. Clin Cancer Res 20: 5349-5358, 2014.

3. McLaughlin-Drubin ME and Münger K: The human papillomavirus E7 oncoprotein. Virology 384: 335-344, 2009. 
4. Scheffner M, Werness BA, Huibregtse JM, Levine AJ and Howley PM: The E6 oncoprotein encoded by human papillomavirus types 16 and 18 promotes the degradation of $\mathrm{p} 53$. Cell 63: $1129-1136,1990$

5. Leal-Garcia M, Garcia-Ortuno L, Ruiz-Azuara L, Gracia-Mora I, Luna-Delvillar J and Sumano H: Assessment of acute respiratory and cardiovascular toxicity of casiopeinas in anaesthetized dogs. Basic Clin Pharmacol Toxicol 101: 151-158, 2007.

6. Chikira M, Tomizawa Y, Fukita D, Sugizaki T, Sugawara N, Yamazaki T, Sasano A, Shindo H, Palaniandavar M and Antholine WE: DNA-fiber EPR study of the orientation of $\mathrm{Cu}(\mathrm{II})$ complexes of 1,10-phenanthroline and its derivatives bound to DNA: Mono(phenanthroline)-copper(II) and its ternary complexes with amino acids. J Inorg Biochem 89: 163-173, 2002.

7. Valencia-Cruz AI, Uribe-Figueroa LI, Galindo-Murillo R, Baca-López K, Gutiérrez AG, Vázquez-Aguirre A, Ruiz-Azuara L, Hernández-Lemus E and Mejía C: Whole genome gene expression analysis reveals casiopeina-induced apoptosis pathways. PLoS One 8: e54664, 2013.

8. Alemón-Medina R, Muñoz-Sánchez JL, Ruiz-Azuara L and Gracia-Mora I: Casiopeina IIgly induced cytotoxicity to HeLa cells depletes the levels of reduced glutathione and is prevented by dimethyl sulfoxide. Toxicol In Vitro 22: 710-715, 2008.

9. Winegarden N: Microarrays in cancer: Moving from hype to clinical reality. Lancet 362: 1428, 2003.

10. Brown H: The real value of microarray technology. Lancet Oncol 4: 326, 2003.

11. Butte A: The use and analysis of microarray data. Nat Rev Drug Discov 1: 951-960, 2002.

12. Jin X, Chen X, Hu Y, Ying F, Zou R, Lin F, Shi Z, Zhu X, Yan X, Li S and Zhu H: LncRNA-TCONS_00026907 is involved in the progression and prognosis of cervical cancer through inhibiting miR-143-5p. Cancer Med 6: 1409-1423, 2017.

13. Xia YF, Pei GH, Wang N, Che YC, Yu FS, Yin FF, Liu HX, Luo B and Wang YK: miR-3156-3p is downregulated in HPV-positive cervical cancer and performs as a tumor-suppressive miRNA. Virol J 14: 20, 2017.

14. Li X, Tian R, Gao H, Yang Y, Williams BRG, Gantier MP, McMillan NAJ, Xu D, Hu Y and Gao Y: Identification of a histone family gene signature for predicting the prognosis of cervical cancer patients. Sci Rep 7: 16495, 2017.

15. Liao XH, Xiang Y, Yu CX, Li JP, Li H, Nie Q, Hu P, Zhou J and Zhang TC: STAT3 is required for MiR-17-5p-mediated sensitization to chemotherapy-induced apoptosis in breast cancer cells. Oncotarget 8: 15763-15774, 2017.

16. Liang B, Li Y and Wang T: A three miRNAs signature predicts survival in cervical cancer using bioinformatics analysis. Sci Rep 7: 5624, 2017.

17. Liang X, Liu Y, Zeng L, Yu C, Hu Z, Zhou Q and Yang Z: miR-101 inhibits the G1-to-S phase transition of cervical cancer cells by targeting Fos. Int J Gynecol Cancer 24: 1165-1172, 2014.

18. Zhao Y, Huang J, Liu T, He S, Shang C, Guo L, Du Q and Yao S: Overexpression of long non-coding RNA RP11-396F22.1 correlates poor prognosis of patients with early-stage cervical cancer. Am J Transl Res 10: 684-695, 2018.

19. Fan Y, Nan Y, Huang J, Zhong H and Zhou W: Up-regulation of inflammation-related LncRNA-IL7R predicts poor clinical outcome in patients with cervical cancer. Biosci Rep 38: pii: BSR20180483, 2018.

20. Ma TT, Zhou LQ, Xia JH, Shen Y, Yan Y and Zhu RH: LncRNA PCAT-1 regulates the proliferation, metastasis and invasion of cervical cancer cells. Eur Rev Med Pharmacol Sci 22: 1907-1913, 2018.

21. Gao D, Zhang Y, Zhu M, Liu S and Wang X: miRNA expression profiles of HPV-infected patients with cervical cancer in the uyghur population in China. PLoS One 11: e0164701, 2016.

22. Jiang L, Shi S, Shi Q, Zhang H, Xia Y and Zhong T: MicroRNA-519d-3p inhibits proliferation and promotes apoptosis by targeting HIF- $2 \alpha$ in cervical cancer under hypoxic conditions. Oncol Res 26: 1055-1062, 2018.

23. Cai N, Hu L, Xie Y, Gao JH, Zhai W, Wang L, Jin QJ, Qin CY and Qiang R: MiR-17-5p promotes cervical cancer cell proliferation and metastasis by targeting transforming growth factor- $\beta$ receptor 2. Eur Rev Med Pharmacol Sci 22: 1899-1906, 2018.

24. Rui X, Xu Y, Jiang X, Ye W, Huang Y and Jiang J: Long non-coding RNA C5orf66-AS1 promotes cell proliferation in cervical cancer by targeting miR-637/RING1 axis. Cell Death Dis 9: $1175,2018$.
25. Liang H, Zhang C, Guan H, Liu J and Cui Y: LncRNA DANCR promotes cervical cancer progression by upregulating ROCK1 via sponging miR-335-5p. J Cell Physiol 234: 7266-7278, 2019.

26. Chen X, Xiong D, Yang H, Ye L, Mei S, Wu J, Chen S, Shang X, Wang $\mathrm{K}$ and Huang L: Long noncoding RNA OPA-interacting protein 5 antisense transcript 1 upregulated SMAD3 expression to contribute to metastasis of cervical cancer by sponging miR-143-3p. J Cell Physiol 234: 5264-5275, 2019.

27. Zheng P, Yin Z, Wu Y, Xu Y, Luo Y and Zhang TC: LncRNA HOTAIR promotes cell migration and invasion by regulating MKL1 via inhibition miR206 expression in HeLa cells. Cell Commun Signal 16: 5, 2018.

28. Feng Y,Zou W, Hu C, Li G, Zhou S, He Y, Ma F, Deng C and Sun L: Modulation of CASC2/miR-21/PTEN pathway sensitizes cervical cancer to cisplatin. Arch Biochem Biophys 623-624: 20-30, 2017.

29. Yang M, Wang M,Li X, Xie Y, Xia X, Tian J,Zhang K and Tang A: Wnt signaling in cervical cancer? J Cancer 9: 1277-1286, 2018.

30. Gupta S, Kumar P and Das BC: HPV: Molecular pathways and targets. Curr Probl Cancer 42: 161-174, 2018.

31. Li P, Hu J, Zhang Y, Li J, Dang Y, Zhang R, Wei L and Shi M: miR-182 promotes cell proliferation of cervical cancer cells by targeting adenomatous polyposis coli (APC) gene. Xi Bao Yu Fen Zi Mian Yi Xue Za Zhi 34: 148-153, 2018 (In Chinese).

32. Smyth GK: Linear models and empirical bayes methods for assessing differential expression in microarray experiments. Stat Appl Genet Mol Biol 3: Article3, 2004.

33. Hollander SL: An unusual case of chronic lymphocytic leukemia. J Med 22: 289-297, 1991.

34. Yu G, Wang LG, Han Y and He QY: ClusterProfiler: An R package for comparing biological themes among gene clusters. OMICS 16: 284-287, 2012.

35. Kanehisa M, Goto S, Sato Y, Furumichi M and Tanabe M: KEGG for integration and interpretation of large-scale molecular data sets. Nucleic Acids Res 40: D109-D114, 2012.

36. Prieto C, Risueno A, Fontanillo C and De las Rivas J: Human gene coexpression landscape: Confident network derived from tissue transcriptomic profiles. PLoS One 3: e3911, 2008.

37. Bravo-Gómez ME, Garcia-Ramos JC, Gracia-Mora I and Ruiz-Azuara L: Antiproliferative activity and QSAR study of copper(II) mixed chelate $[\mathrm{Cu}(\mathrm{N}-\mathrm{N})$ (acetylacetonato)]NO3 and [Cu(N-N)(glycinato)]NO3 complexes, (Casiopeinas). J Inorg Biochem 103: 299-309, 2009.

38. Alemon-Medina R, Bravo-Gomez ME, Gracia-Mora MI and Ruiz-Azuara L: Comparison between the antiproliferative effect and intracellular glutathione depletion induced by Casiopeina IIgly and cisplatin in murine melanoma B16 cells. Toxicol In Vitro 25: 868-873, 2011.

39. Richter K, Haslbeck M and Buchner J: The heat shock response: Life on the verge of death. Mol Cell 40: 253-266, 2010.

40. Isserlin R, Merico D, Voisin V and Bader GD: Enrichment Map-a Cytoscape app to visualize and explore OMICs pathway enrichment results. F1000Res 3: 141, 2014.

41. Marin-Hernandez A, Gracia-Mora I, Ruiz-Ramirez L and Moreno-Sanchez R: Toxic effects of copper-based antineoplastic drugs (Casiopeinas) on mitochondrial functions. Biochem Pharmacol 65: 1979-1989, 2003.

42. Correia I, Borovic S, Cavaco I, Matos CP, Roy S, Santos HM, Fernandes L, Capelo JL, Ruiz-Azuara L and Pessoa JC: Evaluation of the binding of four anti-tumor Casiopeínas ${ }^{\circledR}$ to human serum albumin. J Inorg Biochem 175: 284-297, 2017.

43. Zhang W and Liu HT: MAPK signal pathways in the regulation of cell proliferation in mammalian cells. Cell Res 12: 9-18, 2002.

44. Trejo-Solis C, Palencia G, Zuniga S, Rodríguez-Ropon A Osorio-Rico L, Luvia ST, Gracia-Mora I, Marquez-Rosado L, Sánchez A, Moreno-García ME, et al: Cas IIgly induces apoptosis in glioma C6 cells in vitro and in vivo through caspase-dependent and caspase-independent mechanisms. Neoplasia 7: 563-574, 2005.

45. De Vizcaya-Ruiz A, Rivero-Muller A, Ruiz-Ramirez L, Kass GE, Kelland LR, Orr RM and Dobrota M: Induction of apoptosis by a novel copper-based anticancer compound, casiopeina II, in L1210 murine leukaemia and $\mathrm{CH} 1$ human ovarian carcinoma cells. Toxicol In Vitro 14: 1-5, 2000.

46. Rivero-Muller A, De Vizcaya-Ruiz A, Plant N, Ruiz L and Dobrota M: Mixed chelate copper complex, Casiopeina IIgly, binds and degrades nucleic acids: A mechanism of cytotoxicity. Chem Biol Interact 165: 189-199, 2007.

47. Xia C, Liang S, He Z, Zhu X, Chen R and Chen J: Metformin, a first-line drug for type 2 diabetes mellitus, disrupts the MALAT1/miR-142-3p sponge to decrease invasion and migration in cervical cancer cells. Eur J Pharmacol 830: 59-67, 2018. 
48. Ji Q, Zhang L, Liu X, Zhou L, Wang W, Han Z, Sui H, Tang Y, Wang $Y$ and Liu N, et al: Long non-coding RNA MALAT1 promotes tumour growth and metastasis in colorectal cancer through binding to SFPQ and releasing oncogene PTBP2 from SFPQ/PTBP2 complex. Br J Cancer 111: 736-748, 2014.

49. Dong Y, Liang G, Yuan B, Yang C, Gao R and Zhou X: MALAT1 promotes the proliferation and metastasis of osteosarcoma cells by activating the PI3K/Akt pathway. Tumour Biol 36: 1477-1486, 2015.

50. Xiao H, Tang K, Liu P, Chen K, Hu J, Zeng J, Xiao W, Yu G, Yao $\mathrm{W}$, Zhou $\mathrm{H}$, et al: LncRNA MALAT1 functions as a competing endogenous RNA to regulate ZEB2 expression by sponging miR-200s in clear cell kidney carcinoma. Oncotarget 6 : 38005-38015, 2015.

51. Zhang HM, Yang FQ, Chen SJ, Che J and Zheng JH: Upregulation of long non-coding RNA MALAT1 correlates with tumor progression and poor prognosis in clear cell renal cell carcinoma. Tumour Biol 36: 2947-2955, 2015.

52. Li C, Chen H, Hu L, Xing Y, Sasaki T, Villosis MF, Li J, Nishita M, Minami Y and Minoo P: Ror2 modulates the canonical Wnt signaling in lung epithelial cells through cooperation with Fzd2. BMC Mol Biol 9: 11, 2008.

53. Bian Y, Chang X, Liao Y, Wang J, Li Y, Wang K and Wan X: Promotion of epithelial-mesenchymal transition by Frizzled 2 is involved in the metastasis of endometrial cancer. Oncol Rep 36 803-810, 2016.

54. Zhang E, Li Z, Xu Z, Duan W, Sun C and Lu L: Frizzled2 mediates the migration and invasion of human oral squamous cell carcinoma cells through the regulation of the signal transducer and activator of transcription-3 signaling pathway. Oncol Rep 34: 3061-3067, 2015.

55. Ding LC, Huang XY, Zheng FF, Xie J, She L, Feng Y, Su BH, Zheng DL and Lu YG: FZD2 inhibits the cell growth and migration of salivary adenoid cystic carcinomas. Oncol Rep 35: 1006-1012, 2016.

56. Garzon R and Croce CM: MicroRNAs in normal and malignant hematopoiesis. Curr Opin Hematol 15: 352-358, 2008.

57. Wei Q, Li YX, Liu M, Li X and Tang H: MiR-17-5p targets TP53INP1 and regulates cell proliferation and apoptosis of cervical cancer cells. IUBMB Life 64: 697-704, 2012.

58. Hu J, Wang Z, Shan Y, Pan Y, Ma J and Jia L: Long non-coding RNA HOTAIR promotes osteoarthritis progression via miR-17-5p/FUT2/ß-catenin axis. Cell Death Dis 9: 711, 2018.
59. He Z, Liao Z, Chen S, Li B, Yu Z, Luo G, Yang L, Zeng C and Li Y: Downregulated miR-17, miR-29c, miR-92a and miR-214 may be related to BCL11B overexpression in T cell acute lymphoblastic leukemia. Asia Pac J Clin Oncol 14: e259-e265, 2018.

60. Li Z, Lu J, Sun M, Mi S, Zhang H, Luo RT, Chen P, Wang Y, Yan M, Qian Z, et al: Distinct microRNA expression profiles in acute myeloid leukemia with common translocations. Proc Natl Acad Sci USA 105: 15535-15540, 2008

61. Liu S, Song L, Zeng S and Zhang L: MALAT1-miR-124-RBG2 axis is involved in growth and invasion of HR-HPV-positive cervical cancer cells. Tumour Biol 37: 633-640, 2016.

62. Chen W, Zhao W, Zhang L, Wang L, Wang J, Wan Z, Hong Y and Yu L: MALAT1-miR-101-SOX9 feedback loop modulates the chemo-resistance of lung cancer cell to DDP via Wnt signaling pathway. Oncotarget 8: 94317-94329, 2017.

63. Chen W, Xu XK, Li JL, Kong KK, Li H, Chen C, He J, Wang F, Li P, Ge XS and Li FC: MALAT1 is a prognostic factor in glioblastoma multiforme and induces chemoresistance to temozolomide through suppressing miR-203 and promoting thymidylate synthase expression. Oncotarget 8: 22783-22799, 2017.

64. Pećina-Slaus N: Wnt signal transduction pathway and apoptosis: A review. Cancer Cell Int 10: 22, 2010.

65. Chen S, Guttridge DC, You Z, Zhang Z, Fribley A, Mayo MW, Kitajewski J and Wang CY: Wnt-1 signaling inhibits apoptosis by activating beta-catenin/T cell factor-mediated transcription. J Cell Biol 152: 87-96, 2001.

66. You L, He B, Uematsu K, Xu Z, Mazieres J, Lee A, McCormick F and Jablons DM: Inhibition of Wnt-1 signaling induces apoptosis in beta-catenin-deficient mesothelioma cells. Cancer Res 64: 3474-3478, 2004

67. Li F, Chong ZZ and Maiese K: Winding through the WNT pathway during cellular development and demise. Histol Histopathol 21: 103-124, 2006.

68. Uematsu K, He B, You L, Xu Z, McCormick F and Jablons DM: Activation of the Wnt pathway in non small cell lung cancer: Evidence of dishevelled overexpression. Oncogene 22: 7218-7221, 2003.

This work is licensed under a Creative Commons Attribution-NonCommercial-NoDerivatives 4.0 International (CC BY-NC-ND 4.0) License. 